\title{
Gap Nanoantennas toward Molecular Plasmonic Devices
}

\author{
Aude L. Lereu, ${ }^{1}$ Jacob P. Hoogenboom, ${ }^{2}$ and Niek F. van Hulst ${ }^{3,4}$ \\ ${ }^{1}$ CINaM-CNRS, Campus de Luminy, 13288 Marseille, France \\ ${ }^{2}$ Department of Imaging Science and Technology, Faculty of Applied Sciences, Delft University of Technology, Lorentzweg 1, \\ 2628 CJ Delft, The Netherlands \\ ${ }^{3}$ ICFO_Institut de Ciències Fotòniques, Mediterranean Technology Park, Castelldefels, 08860 Barcelona, Spain \\ ${ }^{4}$ ICREA-Institucío Catalana de Recerca i Estudis Avançats, 08015 Barcelona, Spain
}

Correspondence should be addressed to Aude L. Lereu, lereu@cinam.univ-mrs.fr

Received 30 September 2011; Revised 16 December 2011; Accepted 16 December 2011

Academic Editor: Ali Passian

Copyright (C) 2012 Aude L. Lereu et al. This is an open access article distributed under the Creative Commons Attribution License, which permits unrestricted use, distribution, and reproduction in any medium, provided the original work is properly cited.

\begin{abstract}
Recently we have demonstrated that single fluorescent molecules can be used as non-perturbative vectorial probes of the local field. Here, we expand on such experiments exploiting fluorescence lifetime of single molecules to probe various types of gap nanoantennas. First, studies of the nanoantennas are carried out to evaluate the electric field. We then investigate hybrid systems composed by nanoantennas and randomly positioned fluorescent molecules. Finally, we present a fabrication scheme for the controlled placement of fluorescent molecules at welldefined positions with respect to the dimer nanoantenna, which is a more direct route to probe the local field in an a priori determined way.
\end{abstract}

\section{Introduction to Gap Nanoantennas: Dimers and Nanorods}

Metallic nanoantennas (also called optical antennas) have been, over the past ten years, at the heart of numerous investigations ([1-7] and references therein) due to their optical properties, like strong enhancement and subwavelength confinement of the electrical field [8-17]. The coupling between photons and electrons in these nano-objects is the origin of their extraordinary optical properties. In such 3D structures, the electrons oscillate with (spatial) periods smaller than the wavelength of the incident photons, raising plasmon resonances. Such resonances can then give rise to a strongly localized field enhancement. In addition, nanoantennas permit to couple propagative optical waves into evanescent waves and vice versa. They are therefore promising as intermediary between nanoscale optical and electronic devices and optical fields. Their applications include different fields such as nanoscale imaging and spectroscopy [18-25], light-emitting devices [26-31], photovoltaics [32-41], microfluidics [42, $43]$, and metamaterials in the infrared [44, 45].

Here, we concentrate on gap nanoantennas that can be defined as two symmetric metallic particles (of specific shape and size) separated by a fabricated gap ranging from no gap up to a few tenths of nanometers. In the particular case of gap nanoantennas, the field enhancement and resonance modes have been theoretically predicted [ $46-$ $57]$ and experimentally studied [8-17, 58-64]. Numerous works have been reported on luminescence spectroscopy of gold nanoparticles such as nanorods $[9,10,58]$ and dimers $[12,57,60,61]$ (see Section 3). Two-photon luminescence (TPL) has also been employed and proven particularly sensitive to local fields near metallic nano-objects [12, 58]. Moreover, TPL spectroscopy permits to measure the plasmon resonances of such nanostructures by evaluating the TPL as a function of excitation wavelengths $[61,62]$. Near-field scanning optical microscopy (NSOM) is also extensively used to explore such nano-objects either by locally mapping the field enhancements $[9,10,25,63,64]$ or by grafting an individual nanoantenna at the apex of an NSOM probe [65-70]. However, in these studies, the influence of the near-field probe onto the nanostructures can induce a complex coupling response which has to be taken into account. In particular, plasmonics mode detailed mapping of nanoantennas has been achieved with "apertureless" NSOM using Si-tips [71-74]. Other studies have also looked 
at the fundamental understanding of resonant processes in such nanostructures by means of Raman imaging [75-77] or nonlinear effects [78]. In parallel, analytical and numerical models have been developed in an approach to unify the experimental observations [46-54].

Finally, one alternative approach to study nanoantennas, is to probe their near fields using single molecules (SM) [7981]. By doing so, one can access the local mapping of the nanoantenna fields in a nonperturbative way. Furthermore, integrating SM together with metallic nanoantennas is an ultimate step in terms of applications for the technological miniaturization drive. In most cases, the functionality of SM is inherently defined by the electronic or optical properties of the molecules. However, the construction of active molecular-scale devices often requires embedding the molecule in a nanostructured environment that provides a means to address, tailor, or control the molecular functionality. Gap nanoantennas are particularly promising interfaces, because of their strongly localized electromagnetic field modes, both in the spatial and the frequency domain. Consequently, the emerging field of molecular plasmonics [82] holds great promise for applications in areas like sensing, light harvesting and energy conversion, single-photon sources, all-optical components, and electronic-optical interfacing. However, a major task remains in the mutually well-defined positioning of both functional molecules and metallic nanostructures with nanometer-scale precision. Different approaches have been developed relying on random deposition of functional molecules, for example, by spincoating [83], bulk overcoating of a metallic structure [84], or plasmonic micropositioning $[42,43]$. In highly symmetric nanoparticle systems, one degree of positional control has been achieved by employing a spacer layer that can be functionalized [85-87]. The full exploitation of molecular functionality requires localized and designed molecular positioning at arbitrary locations with respect to a tailored plasmonic nanostructure. Recently, encouraging progress in this direction has been made by double e-beam lithography, positioning a single quantum dot on the feed element of an array antenna [88].

Here, we will describe the full characterization of antennas needed to realize resonant nanoantenna structures overcoated with low ("single molecule") concentrations of fluorescent molecules. This characterization serves to extract the individual optical response of the nanoantennas in an effort to match the fluorescence spectra of molecules. Parts of this work have been described previously [63, 64, 79]. After these introductory results, we will present fluorescence measurements on nanoantennas overcoated with ultralow concentrations of molecules. We will address measurement of the fluorescence lifetime, to map the local density of states around the nanoantennas [80], in direct competition with the intrinsic luminescence of the antennas. Finally, a fabrication procedure that allows for the controlled positioning of molecules with respect to the antenna, together with initial related results, will be presented.

The subject matter is divided over the various sections in the following way: in Section 2, we introduce the nanoantenna samples (dimer and nanorod arrays), explaining the fabrication process and characterizing their spectroscopic response. In Section 3, we describe one photon luminescence of dimer antennas. These first two sections lay the basis to first evaluate the optical response of the nanoantennas, without (dye) molecules but under excitation conditions typical for single-molecule spectroscopy. In Section 4, we discuss the response of randomly scattered single molecules over dimers. Finally, molecular probing will be refined using a well-controlled molecular probing in Section 5. Section 6 gathers our conclusions.

\section{Nanofabrication and Spectroscopic Insights of Gold Dimers and Nanorods Arrays}

The nanoantenna electromagnetic response is known to depend highly on size, geometry, and even chemical treatment. A large panel of sizes, shapes, and compositions has therefore been fabricated at the nanoscale either by bottom-up colloidal chemistry [89-94] or by top-down nanofabrication techniques $[11,61-64,95,96]$ with the use of electron beam lithography or ion beam milling. A multitude of shapes is emerging offering a wide range of field pattern and strength. Here, we will report on dimer and nanorod-like structures.

The parameters specifying the geometry of the dimer and nanorod-like structures are the aspect ratio (AR), defined as the ratio of length to width, the gap separation distance between the two nanoparticles constituting the gap nanoantenna, and the thickness. The single nanoantennas in these studies have an AR in the range from 1.0 (dimers) to 4.5 (nanorods), as illustrated in insets of Figure 1. We mainly kept the width around $100 \mathrm{~nm}$ and varied the length of the structures. The different fabrication steps are depicted in Figure 1. We used conventional e-beam lithography (FEIQUANTA 200 electron microscope associated with a Raith ELPHY) and metal depositions followed by a lift-off process to realize periodic arrays $(100 \times 100 \mu \mathrm{m})$ of nanoantennas. Prior to any investigation, we calculated the spectroscopic response (in the excitation wavelength range of $400 \mathrm{~nm}$ to $700 \mathrm{~nm}$ ) for the given geometry and performed far-field spectroscopic measurements on the arrays of fabricated nanostructures. This is illustrated in Figure 2 for the dimers and Figure 3 for the nanorods.

In this section, we focus on and quantify the different resonant modes in the visible, a spectral range spanned by our excitation sources. Therefore, we carried out local spectroscopic measurements on distinct arrays of nanoantennas, under white light illumination and two orthogonal linear polarization states. Throughout this paper, the ppolarization state will be referred to as the polarization excitation along the long axis of the structures, whereas the s-polarization state will be associated with the perpendicular one. In parallel, we have calculated the specific spectral response at the gap region of an individual pair of nanoantennas for each involved AR. While the presented $2 \mathrm{D}$ simulations (insets of Figures 2 and 3) seek to illustrate the overall field distribution under defined excitation conditions (polarization state and excitation wavelength), it is not to be directly confronted to the measurements. 


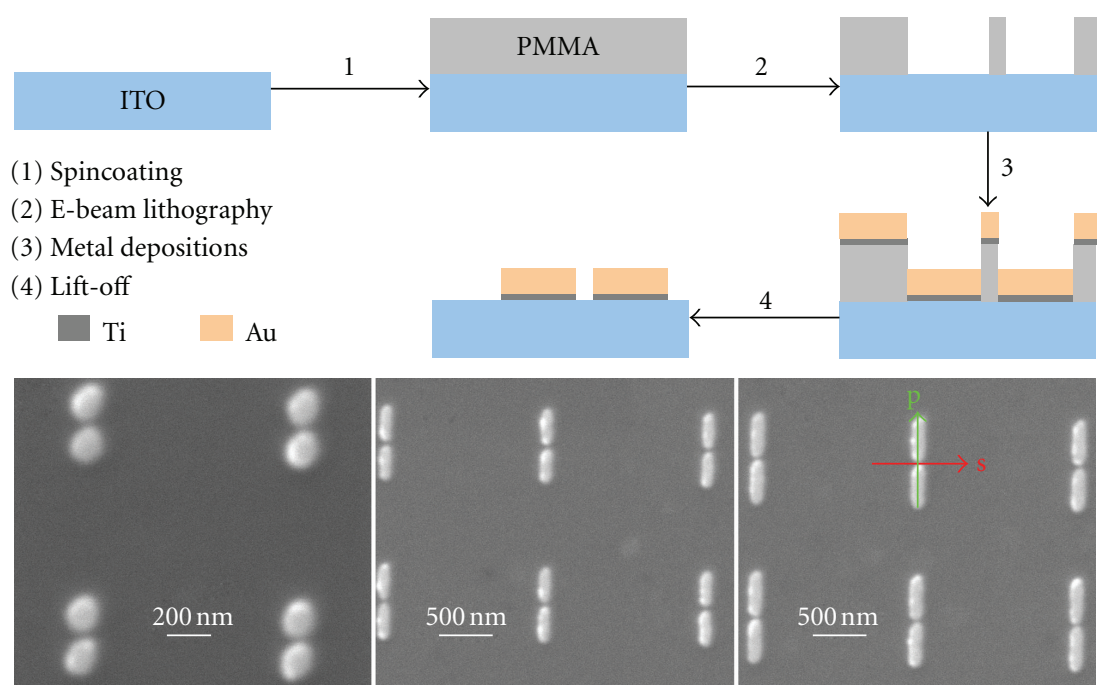

(a)

(b)

(c)

FIGURE 1: Fabrication steps of nanoantenna arrays using e-beam lithography and metal depositions. SEM images of selected nanoantenna arrays, used in the reported studies, are presented in (a) for dimers, and in (b) and (c) for nanorods with different lengths and therefore different ARs. The arrows in (c) define the used excitation polarizations, with p-polarization along the long axis of the nanostructures and $s$ perpendicular to the long axis.

In Figure 2, the measured scattering spectra of dimers, for both excitation s and p-polarization states, and the calculated spectral response of an individual dimer are displayed. Under p-polarization excitation, the measured peak is close to $635 \mathrm{~nm}$, while the calculation presents a peak at around $655 \mathrm{~nm}$. This experimental blue shift may be explained both by geometrical variations occurring during the fabrication process and by substrate effects. The numerical work was carried out for a single dimer in free standing space. Under s-polarization excitation (electric field perpendicular to the dimer axis), a very weak peak centered on $599 \mathrm{~nm}$ is observed. For this wavelength, the simulation does not present any peak as the field distribution is mainly located at the edges of the dimers and not in the gap from where the calculated spectrum is extracted. Finally, secondary peaks are predicted at around $500 \mathrm{~nm}$ for both polarization states but were not experimentally observed. Neglecting the effect of the substrate as well as retardation effects (more pronounced for shorter wavelengths) in the simulation may explain the discrepancy over these auxiliary peaks.

In Figure 3, theoretical spectra are presented for nanorods for each excitation polarization state, both in the nonretarded (continuous lines of Figures 3(a) and 3(b)) and the retarded regime (Figures $3(\mathrm{c})$ and $3(\mathrm{~d})$ ). In the retarded regime, the results are the solutions of the wave equation, whereas in the nonretarded regime, the time dependence has been neglected. The experimental spectra are included in Figures 3(a) and 3(b) (dashed lines). Note that the different nanorods' lengths are highlighted in the colors of the curves, red for the $150 \mathrm{~nm}$ long, blue for the $350 \mathrm{~nm}$, and green for the $450 \mathrm{~nm}$. For the p-polarization state, a 2- to 4-fold enhancement, defined as the ratio of the electric field in the presence and in the absence of the nanoantennas, is reached, both theoretically (in the retarded regime) and experimentally. The theoretical values obtained in the quasistatic regime are much higher, apart for the case of a nanoantenna with an AR of 1.5. This is to be expected as the time dependence becomes more important for structures with increasing sizes where the distances can be larger than the excitation wavelength. This is actually illustrated in Figure 3 where the two models can be directly compared. One can see that for the $150 \mathrm{~nm}$ long rods the quasistatic and retarded responses are similar, whereas for the $450 \mathrm{~nm}$ long rods, this does not hold anymore. Furthermore, if we now compare simulations in the retarded regime with the experiment, we see that the experimentally observed intensities are mainly higher than the simulated ones. This observation can also be rationalized by the contribution of the additional field imaged on the edges of the structures during the measurements or in the field distributions. However, the theoretical spectra concentrate only on the electric field in the gap.

Under s-polarized excitation, the gap region, for each considered AR, shows no predicted field enhancement, except in the retarded regime for the $450 \mathrm{~nm}$ long rods in the 465 to $510 \mathrm{~nm}$ spectral range. Indeed, an up to 2 -fold enhancement is then expected. Experimentally, we obtain a resonance peak around $465 \mathrm{~nm}$ for the $450 \mathrm{~nm}$ long rods. For the $350 \mathrm{~nm}$ and $150 \mathrm{~nm}$ long rods, the peaks are centered at $462 \mathrm{~nm}$ and $460 \mathrm{~nm}$, respectively, with corresponding enhancement factors between 2 and 4 . The differences (in terms of peak wavelength and enhancement factor) with the simulation may result from fabrication inhomogeneities and/or from the fact that the experimental values are retrieved after integration of the field in a region encompassing the whole structure instead of the gap alone. This is also predicted as seen in the insets representing the total field distribution at the point B of Figure 3 , where 


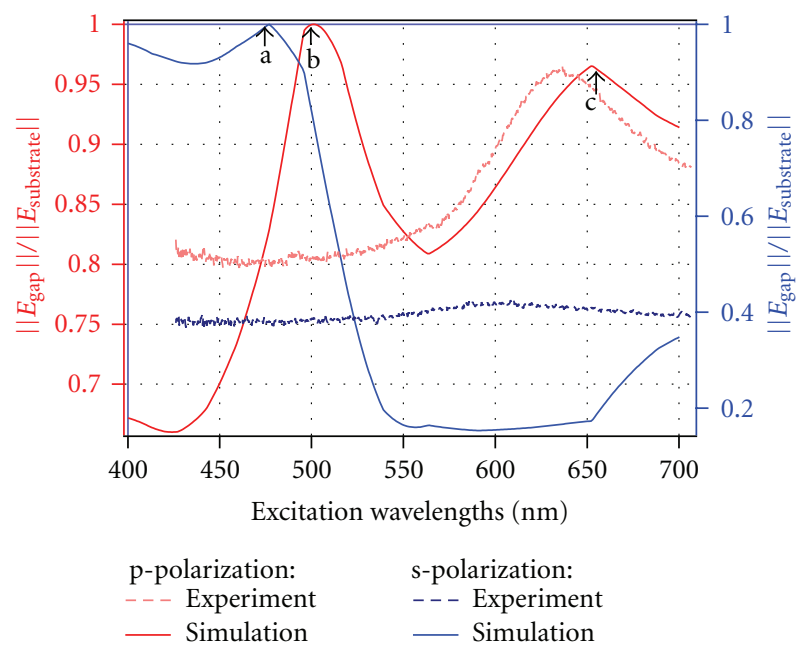

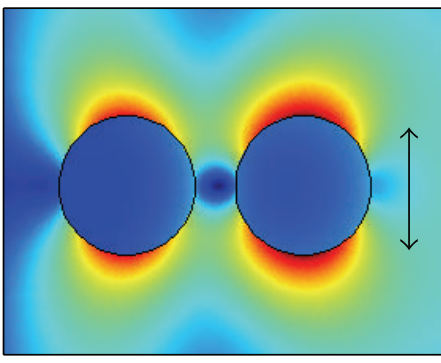

$m=0.3, M=2.1$

(a)
Field distribution at the peaks a, b, and c

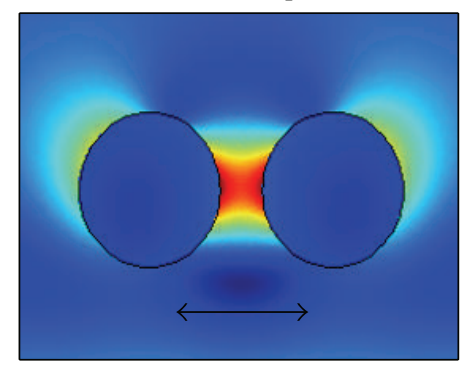

$m=2.9, M=4.37$

(b)

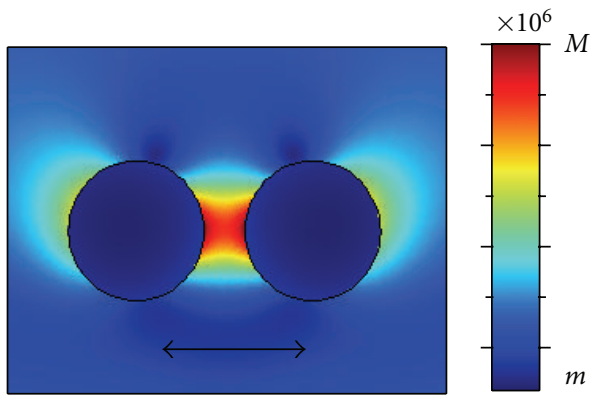

$m=0.1, M=1.1$

(c)

FIGURE 2: Theoretical and experimental spectra of the electric field and extinction for dimers (particles with $100 \mathrm{~nm}$ diameter) with $30 \mathrm{~nm}$ gap. The (a) to (b) labels indicate the excitation parameters where resonances occur. These are then used in the corresponding insets to display the field distribution under these excitation conditions. The insets (a) to (c) depict the total field fingerprint for each spectral peak (black arrows show the polarization state), to illustrate the resonances and local field enhancement around the dimers.

the strongest fields are mainly located at the edges of the structures and not in the gap.

By linking the geometrical parameters of the nanoantennas with their optical responses, the presented spectroscopic studies served as a guide for subsequent experiments. In the following, we will present experiments aimed at mapping the luminescence distribution in the vicinity of the nanoantennas as a function of incident polarization. These experiments will allow acquiring a complementary insight into the optical response of the nanoantennas. The knowledge of predicted electric fields, presented in the insets of Figures 2 and 3, will assist the analysis of the experimental results.

\section{Luminescence of Dimers under Pulsed Excitation}

In this section, we present one-photon excitation photoluminescence measurements on nanoantennas. The influence of the polarization state is explored by illuminating the nanoantennas with both $\mathrm{p}$ and s-polarized light. As already mentioned, p-polarized incident light is polarized along the long axis of the dimmers, whereas s-polarized incident light is polarized perpendicular to the dimer axis. A comparison between an optimal dimer gap of $30 \mathrm{~nm}$ (also referred to as resonant case) and no gap (nonresonant case) is also given.

As mentioned in Section 2, samples were made by e-beam lithography and metal deposition. The sample design consists here of several $100 \mu \mathrm{m} \times 100 \mu \mathrm{m}$ arrays of gold nano-objects forming either a dimer or a single particle (monomer) as illustrated in Figure 4(a). In order to make an unambiguous distinction between dimers and monomers in the optical experiments, the global array was fashioned by alternating three lines of dimers and two of monomers. The diameter was chosen as $100 \mathrm{~nm}$ and the height as $40 \mathrm{~nm}$, for an optimum gap of $30 \mathrm{~nm}$ [23]. Finally, in order to observe any gap effect, similar arrays of dimers with no gap, shown in Figure 4(b), were studied in parallel. The geometric parameters of the gold particles were chosen so that the dimers would be resonant at around $635 \mathrm{~nm}$ (see scattering spectrum in Figure 2). The choice of the $635 \mathrm{~nm}$ resonant wavelength was aimed at matching the absorption spectrum of the molecular species used 


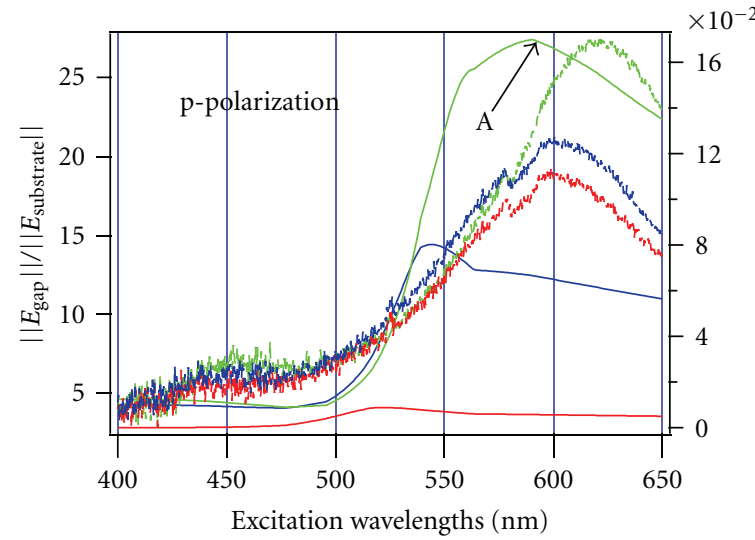

(a)

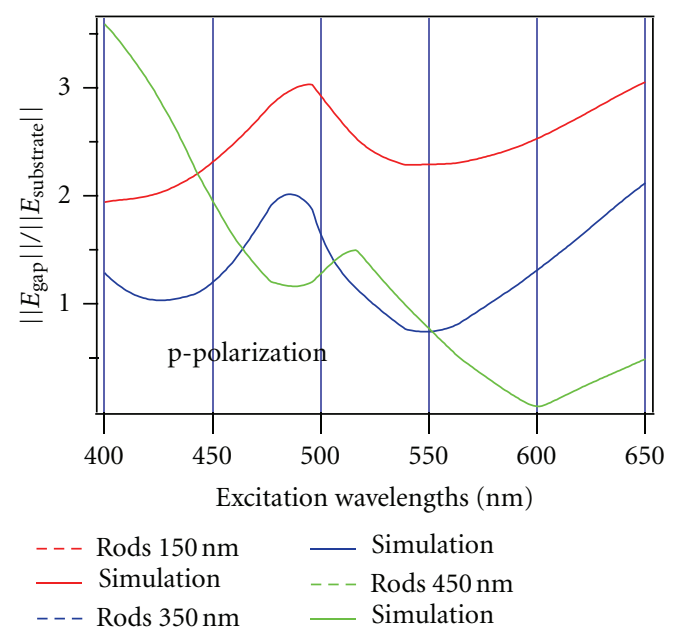

(c)

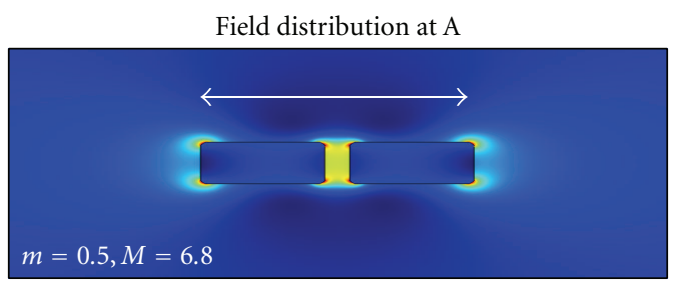

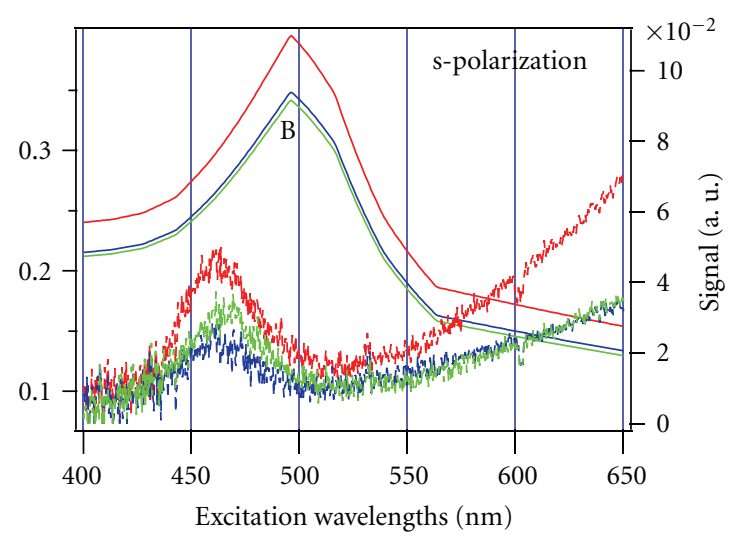

(b)

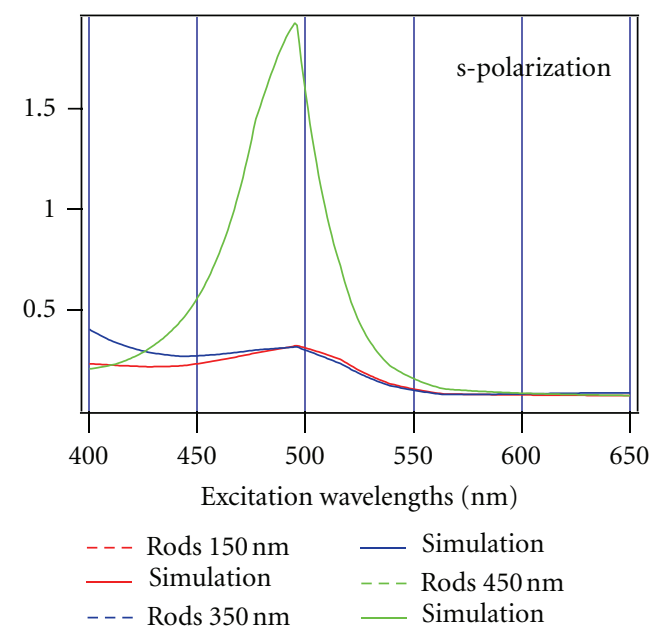

(d)
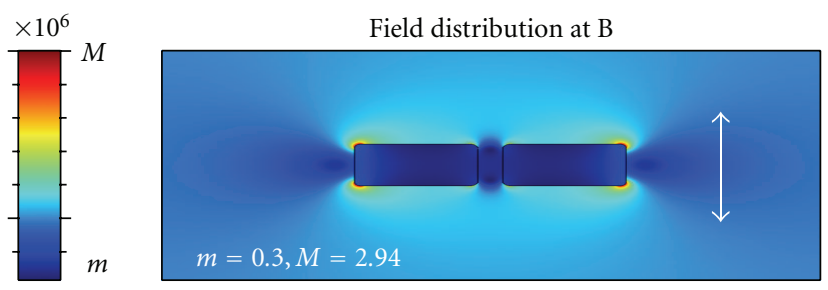

FIGURE 3: Theoretical and experimental spectra of the electric field intensity and extinction for antennas made of nanorods of different lengths and with $30 \mathrm{~nm}$ gap. (a) and (b) Plot the experimental and theoretical spectra (in the quasistatic regime), while (c) and (d) show the theoretical spectra in the retarded regime. All spectra are depicted for both excitation polarization states. Note that the theoretical curves depict the electric field in the gap of the structures, whereas the data represent the response of the total structure. The two insets give the total electric field distribution for the $450 \mathrm{~nm}$ long rods at the peaks A (at $580 \mathrm{~nm}$ ) and B (at $500 \mathrm{~nm}$ ). The white arrows indicate the considered polarization.

as a probe, here $\operatorname{DiD}\left(1,1^{\prime}\right.$-dioctadecyl-3,3,3', $3^{\prime}$-tetramethylindodicarbocyanine), a photostable molecule commonly used in single-molecule studies.

Luminescence measurements on individual dimers (and monomers) were carried out using a confocal microscope, as shown in Figure 5. A confocal scanning fluorescence microscope with a high numerical aperture (1.3 NA) objective was equipped with two avalanche photodiodes (APDs) detecting the respective ( $\mathrm{p} \& \mathrm{~s}$ ) cross-polarizations using a polarizing beam splitter $[63,64]$. The excitation source was chosen to be a picosecond (ps) laser (90 ps pulses at $20 \mathrm{MHz}$ repetition rate, average power density $\sim 34 \mathrm{~kW} / \mathrm{cm}^{2}$ at $\lambda_{\mathrm{exc}}=635 \mathrm{~nm}$ ). The emitted luminescence from the nanostructures (see examples in inset (c) of Figure 5) was collected by the same objective and then directed to the two APDs after discrimination from the excitation light by an appropriate dichroic mirror and a long pass filter. Thereafter, the two luminescence images obtained via the two different 


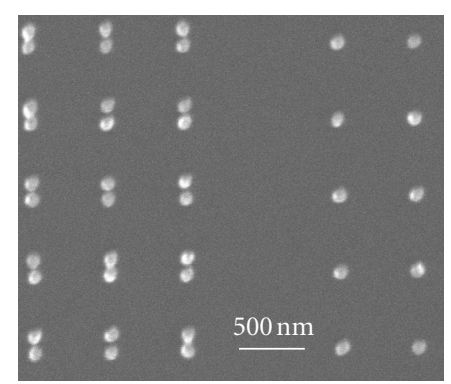

(a)

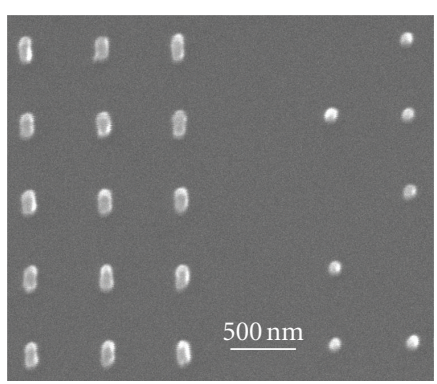

(b)

FIGURE 4: SEM images of the specifically designed samples. Alternations between three lines of the investigated dimers and two lines of reference monomers are made. All nanoparticles are $100 \mathrm{~nm}$ in diameter. In (a), the dimer's separation gap is $30 \mathrm{~nm}$, and in (b) the dimers have no gap (i.e., contact). The mutual distance between two dimers (center to center) is fixed at $600 \mathrm{~nm}$ in both directions.

APDs are recombined to result in a polarization-dependent luminescence image (as insets (d) and (e) of Figure 5). Finally, to simultaneously detect the transmitted excitation light through the sample, a photodiode was mounted on the opposite side of the high NA objective, above the sample plane. An example of such a transmitted image $(10 \times 10 \mu \mathrm{m})$ is given in the inset (a) of Figure 5, where the series of two lines of black dots are associated with the gold monomers, and the series of three black lines are associated with the dimers. The center to center dimer separation of only $600 \mathrm{~nm}$ is only slightly larger than the diffraction limit of the confocal microscope, which prevents from fully resolving two neighboring dimers. As a result, the dimers appear as a semicontinuous black strip with dots in the transmission image. Note that the same experimental setup was utilized as a basis for all the results reported further.

The luminescence (Figure 6 and insets (d) and (e) of Figure 5) is always recorded simultaneously with the transmission signal (inset (a) of Figure 5) to ensure the monomers' positioning with respect to the dimers on the luminescence images. Figure 6 outlines the luminescence images as a function of the excitation polarization and the gap size. The color scale gives the polarization state of the detected luminescence going from green pixels for "pure" p-polarization (i.e., along the dimers axis) to red pixels for "pure" s-polarization. The yellow color represents an equal contribution of both $\mathrm{p}$ - and s-polarization components. From these images, one can extract different aspects. First, the (expected) isotropic emission in polarization of the monomers is clearly highlighted from its yellow appearance (i.e., $\mathrm{p}=\mathrm{s}$ ) on each image. Next, compared to this isotropic emission, the luminescence from the dimers is predominantly p-polarized (green appearance). However, a large variation in luminescence intensity from one nanoantenna to another is observed. This can be mainly explained by the variation in shape and size of each gold particle as introduced by the fabrication process. To take into account the variability between antennas, we made a statistical analysis over the nanoparticles designed to be identical. This statistical analysis serves to study the optical response of metallic structures as a function of fabrication uncertainties.
According to the first qualitative observations on the resonance behavior of such nanoantennas, we oriented the first statistical analysis to extract the peak intensity for each luminescent spot on each image. An intensity distribution is therefore excerpted for the case of a $30 \mathrm{~nm}$ gap, and no gap (see columns in Figure 6), and for p-and s-excitation polarizations (see rows in Figure 6). Similar studies on the monomers served as a reference for polarization effects and allowed the suppression of possible substrate effects. The resulting histograms are depicted in insets $\left(a_{2}-d_{2}\right)$ of Figure 6 for the dimers and insets $\left(a_{3}-d_{3}\right)$ for the monomers. We thus confirmed the isotropic behavior of the monomers, where the polarization ratio (i.e., the ratio of the p-detected over the s-detected polarization) is extracted to be around unity for both $\mathrm{p}$ - and s-polarized excitation.

For the dimers with the two considered gap sizes, the luminescence distribution is shifted toward the higher intensity values for the p-polarized luminescence (with respect to the s-polarized luminescence). This is valid for both excitation polarizations, but the effect is more pronounced for the resonant case (i.e., dimers with a $30 \mathrm{~nm}$ gap) with p-polarized illumination and p-polarized detection. This is expected as the localized plasmon excitation is involved in the process as illustrated, in the insets of Figure 2, by the electric field amplitude in the near field of the dimers. Indeed, for the $30 \mathrm{~nm}$ gap, the p-polarized excitation leads to a strong coupling between the local charges of each gold particle constituting the nanoantenna. By opposition, the local charges of the individual particles are independently excited under s-polarized excitation. For the no gap dimers, we made similar observations. However, the strong coupling for p-polarized excitation is far less pronounced. This is foreseen as the particles do not couple with each other but act as a unique elongated particle.

In a second statistical analysis, we concentrated on the polarization ratio distribution extrapolated from the luminescence peaks in Figure 6. The polarization ratio is defined as the ratio of the p-polarized over the s-polarized luminescence intensity. We seek here to quantify the polarization effect using the polarization ratio. This is illustrated in Figure 7(a) for the $30 \mathrm{~nm}$ gap dimers and (b) for the 


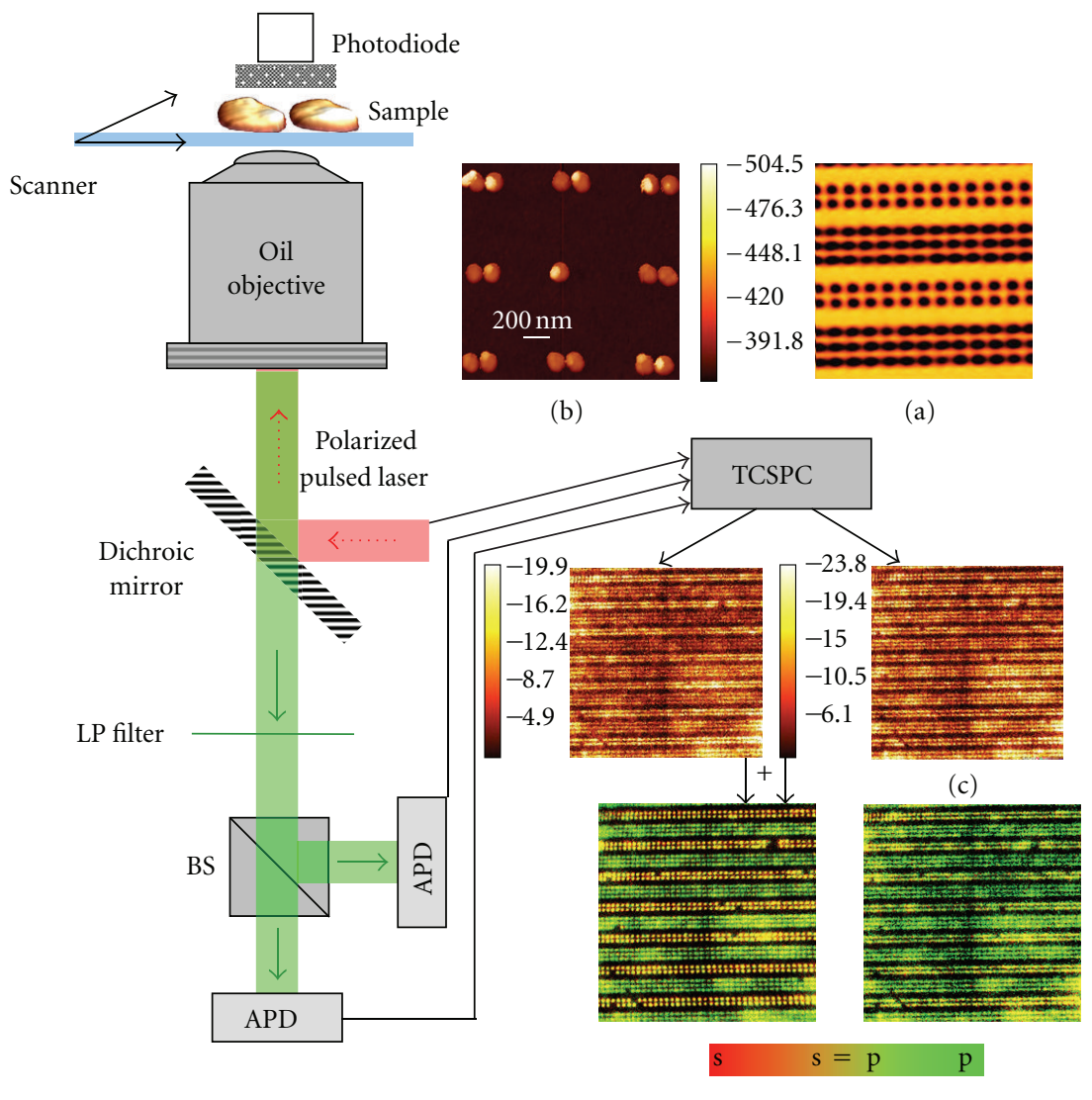

(d)

(e)

Figure 5: Confocal microscope with single-molecule detection capability. A pulsed laser serves as the excitation light incident on the sample via a dichroic mirror and an oil immersed objective (numerical aperture 1.3). The luminescence EPI-generated by the sample is collected through the objective, and then directed through the dichroic mirror and long-pass (LP) filters toward the detectors. The detectors are avalanche photodiodes (APDs), detecting the two crossed-polarization components of the luminescence. These components are formerly separated by a polarizing beam splitter (BS) and recorded via a time-correlated single-photon counting (TCSPC). A photodiode is also placed above the sample, to collect simultaneously the transmitted field as in (a). The piezo scanner, associated with the feedback controller, allows acquiring point-by-point images in the $x y$-plane of the sample, as illustrated by the two images in (c). A composed image from the images in (c) is then generated for p- (inset d) and for s-polarized (inset e) excitation. The inset (b) is a selected AFM image of one of the explored samples.

no-gap ones. In Figure $7(\mathrm{a})$, the polarization ratio ranges between 1.2 and 5.7 for p-polarized excitation and between 1.2 and 2.4 for s-polarized excitation, whereas in Figure 7(b), the polarization ratio ranges between 1.3 and 2.9 for $\mathrm{p}$ and between 1.1 and 2.5 for s-polarized excitation. This leads to an average ratio for the $30 \mathrm{~nm}$ gap of 3.1 for p-polarized excitation against 1.6 for s-polarized excitation, whereas in the no-gap case, the average is 1.8 for p against 1.4 for $\mathrm{s}$.

An averaged 3-fold enhancement is hence shown with respect to the monomers (close to unity, independently of the excitation polarizations), exhibiting a maximum 6fold enhancement, for some of the dimers. This is in agreement with the reported 100-fold enhancement using two-photon luminescence on bowties [11] and the 10-fold enhancement measured in TPL [61]. The weaker increase also observed for the nonresonant case with p-polarized excitation may be attributed to the elongated shape of the dimers. For s-polarized excitation, no noticeable difference is seen with respect to the gap size. This can be explained by the excitation of two independent dipoles that do not interact. Furthermore, this statement is reinforced by the actual measurement of values close to 1 in the s-polarized detected luminescence, approaching the monomer behavior.

To conclude, luminescence of dimer-like nanoantennas was measured under picosecond pulsed excitation at relatively high excitation power compared to typical fluorescence measurements, highlighting (i) the low intrinsic luminescence of the nanoantennas and (ii) the wide distribution from one antenna to another. The effect of excitation polarization and gap size was also investigated. This preliminary study provided insights into understanding the local response of such metallic nanoantennas and may help to understand the nanoantenna-single molecule coupling, as reported below. 


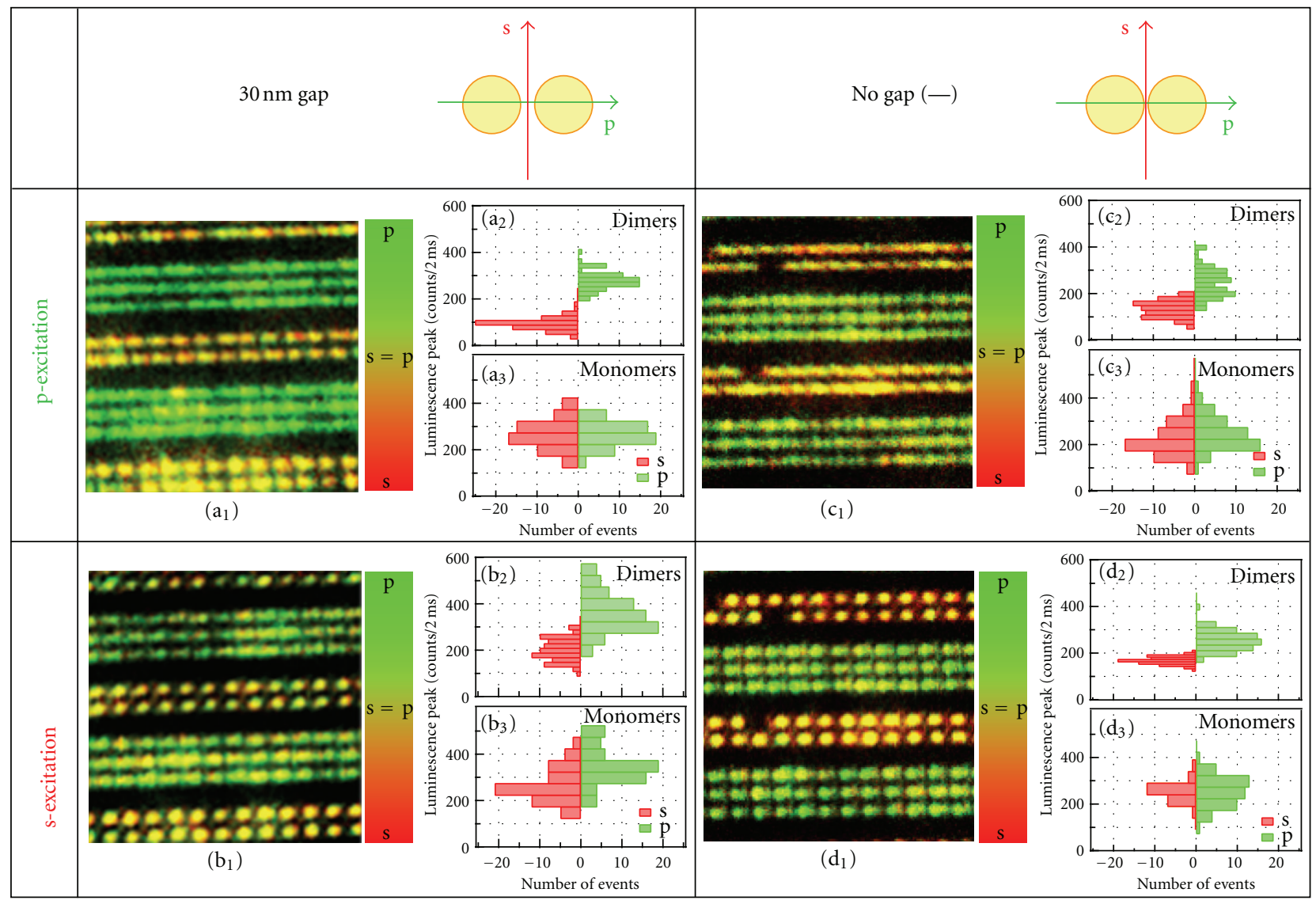

Figure 6: Luminescence measurements of single gold dimers with a gap of $30 \mathrm{~nm}$ ( $\mathrm{a}$ and b) and with no gap (c and d). The figures (a and c) illustrate the luminescence of the structures when illuminated with p-polarized light (i.e., parallel to the long axis of the dimers). Figures ( $b$ and $d$ ) show the same results for s-polarized excitation light (perpendicular to the dimer axis). ( $a_{1}$ to $\left.d_{1}\right)$ Show luminescence images of a $10 \times 10 \mu \mathrm{m}$ scanned area. The color scale gives the polarization state of the detected field. $\left(\mathrm{a}_{2}\right.$ to $\left.\mathrm{d}_{2}\right)$ and $\left(\mathrm{a}_{3}\right.$ to $\left.\mathrm{d}_{3}\right)$ Show distribution of the luminescence peak for dimers (over 60 dimers were considered for each case) and monomers (over 50 monomers were considered for each case), respectively.

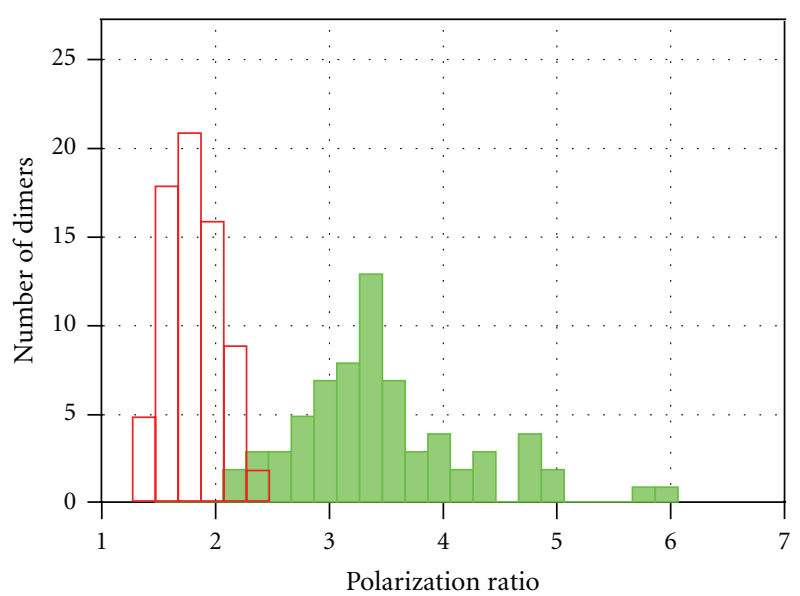

p-excitation $\square$ s-excitation

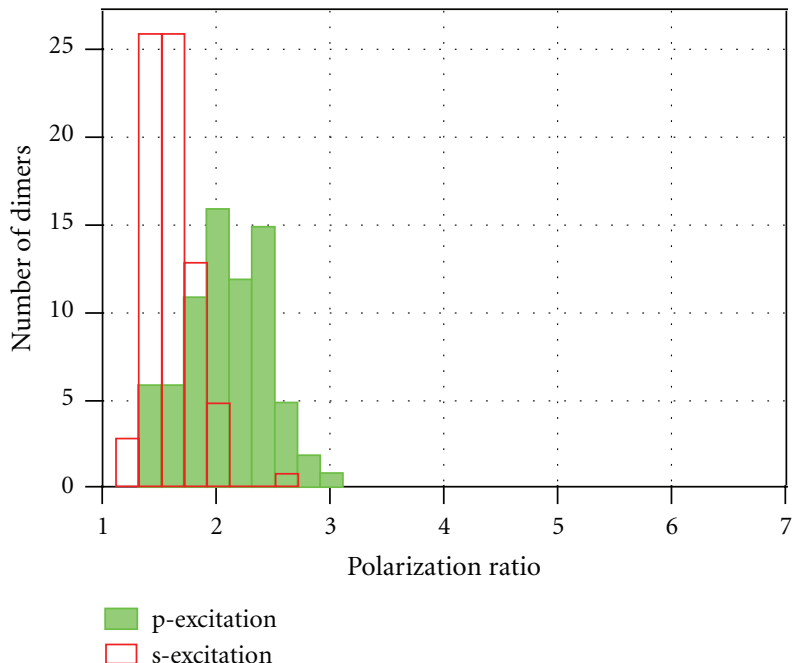

(b)

FIGURE 7: Distribution of the polarization ratio in detection (p-polarized over s-polarized luminescence) for about 60 dimers illuminated under p- or s-polarized light. (a) is for the dimers with $30 \mathrm{~nm}$ gap and (b) for the dimers with no gap. 


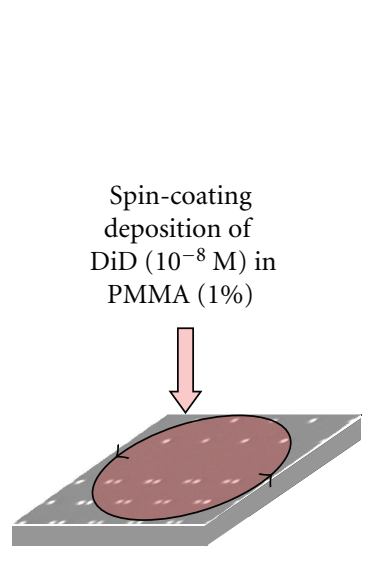

(a)
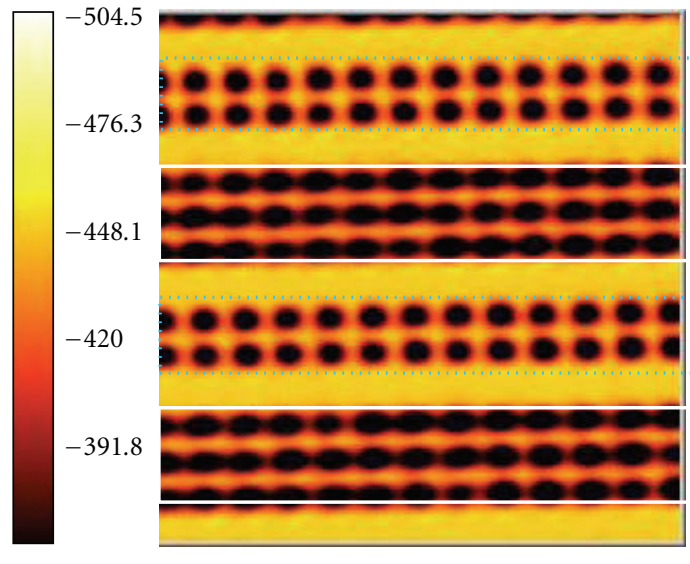

(b)

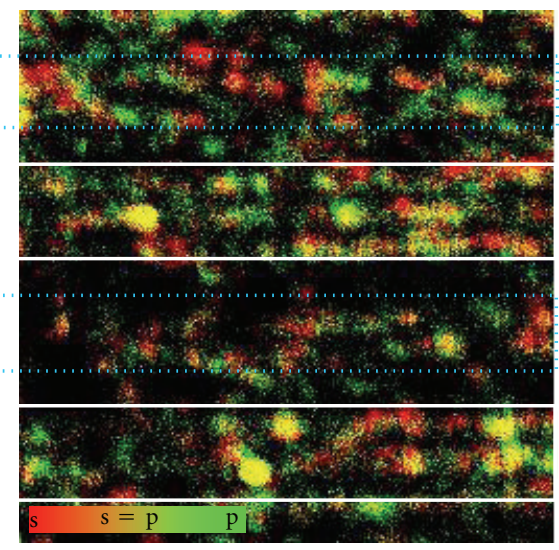

(c)

Figure 8: (a) Scheme of a random deposition of molecules over samples as described in Figure 4(a). (b) Optical transmission microscopy image and (c) fluorescence microscopy image. The white and blue squares highlight the dimer and monomer regions, respectively. The color scale in (c) indicates the polarization as given in Figure 6.

\section{Fluorescence of Molecules at Metallic Nanoantennas}

After imaging the luminescence of individual nanoantennas, we exploit single fluorescent molecules as nonperturbative vectorial point detectors to map the local photonic density of states, with nanometric precision [97]. We have previously demonstrated this procedure for a metal Fabry-Pérot-like cavity [80]. In that study, the measured spatial fluorescence lifetime variations were shown to map the local density of states inside the cavity, provided intrinsic molecular lifetime variations were taken into account. Hereto, individual fluorophores are randomly spread over the sample and are studied in terms of molecular position, orientation, and fluorescence brightness.

As already mentioned in Section 2, the plasmon resonances in metallic nanoantennas are associated with an absorption peak and a localized field enhancement. The proximity of a metallic nanoantenna or nanostructured film is likely to affect the excitation and emission processes of a fluorophore, especially under the plasmon resonance conditions.

In this section, we use the fluorophore properties to probe the local field of the metallic gap nanoantennas. The optical characterization was carried out using the same home-built inverted confocal microscopy setup with singlemolecule sensitivity described in Section 3. The samples were excited using a pulsed laser $(90 \mathrm{ps}, 20 \mathrm{MHz}$ repetition rate, $1 \mathrm{~kW} / \mathrm{cm}^{2}$ ) at a wavelength of $635 \mathrm{~nm}$, optimum to the excitation spectrum of the fluorophore and at a power considerably lower than in Section 3. The collected fluorescence light was separated from the excitation beam using a dichroic mirror and a long-pass filter assembly and subsequently split into two orthogonal polarization directions, each of which was focused onto the detection area of an APD. Transmitted light was simultaneously detected using a photodiode to identify the dimer series from the monomers as in Section 3 and Figure $8(\mathrm{~b})$. On the previously studied nanoantennas arrays, a solution of $\mathrm{DiD}\left(1,1^{\prime}\right.$-dioctadecyl-3,3,3', $3^{\prime}$ tetramethylindodicarbocyanine) molecules in a PMMA (polymethyl methacrylate) matrix was then spin-coated over the nanoantennas, as seen in Figure 8(a). In the intermediate regions with no antenna in Figure 8(c), the fluorophore emission was evaluated to be at the single-molecule detection level as expected. In the regions where nanoantennas are present, one can observe higher emission intensity. Furthermore, in the dimer regions, the average emission intensity is even higher than in the monomer regions (see Figure $8(\mathrm{c})$ ). This may be due to an enhancement of singlemolecule excitation and emission by the antenna. However, the gold luminescence signal, despite the weak excitation power (compared to the excitation power used in Section 3 ), may be detectable, or the random deposition of the single molecules is not homogeneous because of the topographic presence of nanoantennas.

In order to investigate the separate contributions of dimer nanoantenna intrinsic luminescence and molecular fluorescence in more detail, we turned to a perylene dye that we used in previous studies because of its excellent photostability [98, 99]. This dye allows for observation times up to minutes and in addition shows an on-off switching (blinking) on timescales of seconds [99] which facilitates measuring the separate responses. In order to match the nanoantenna response to the greener (compared to DiD) wavelength spectrum of the perylene dye (excitation around $565 \mathrm{~nm}$ ), the thickness of the nanoantenna dimers was increased to $60 \mathrm{~nm}$. This shifts the dimer resonance spectrum to the same wavelength range as the perylene fluorescence. We spin-coated a $50 \mathrm{~nm}$ layer of perylene in PMMA over the substrate containing the antennas. Samples were then excited using pulsed (280 fs, $80 \mathrm{MHz}$ repetition rate) laser illumination (Coherent Mira 900-F pumped by a Coherent Verdi V-18, with an APE optical parametric oscillator and an APE pulse picker set at a 1 in 20 picking 

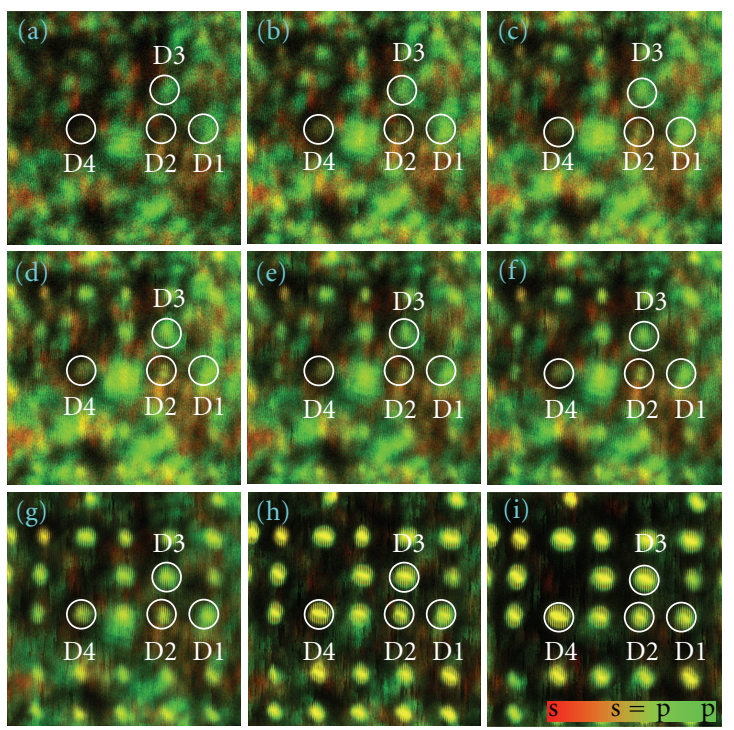

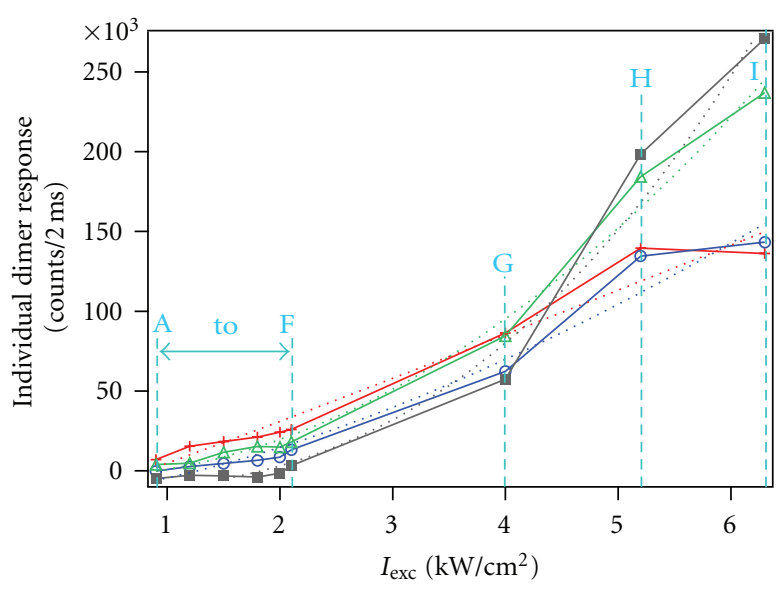

\begin{tabular}{rlr} 
Experiment & \multicolumn{2}{l}{ Fitted $\left(I_{\text {exc }}\right)^{m}$} \\
$\square$ D1 & $\cdots \cdots$ & $m=1.05$ \\
$\square$ D2 & $\cdots \cdots$ & $m=1.53$ \\
$\square$ D3 & $\cdots \cdots$ & $m=2.01$ \\
$\square$ D4 & $\cdots \cdots$ & $m=2.54$
\end{tabular}

FIGURE 9: Femtosecond pulsed excitation over gold dimers. The excitation wavelength is fixed at $565 \mathrm{~nm}$, and its power density is varied from 0.9 (a) to $6.3 \mathrm{~kW} / \mathrm{cm}^{2}$ (i). The dedicated sample here is a $100 \times 100 \mu \mathrm{m}$ array of dimers with a $100 \mathrm{~nm}$ diameter, a $30 \mathrm{~nm}$ gap, and a $60 \mathrm{~nm}$ thickness, that matches the excitation wavelength. A $50 \mathrm{~nm}$ layer of perylene in PMMA is spin-coated over the gold dimers as in Figure 4(a). The graph displays the individual response of selected dimers (circled on the image series) as a function of the excitation power. From one dimer to another, one can see various power dependences of the signal, going from close to linear behavior (for D1) to overquadratic one (in D3 and D4).

ratio) and with circular polarization. As described above, the collected fluorescence light was separated from the excitation beam using a dichroic mirror and a $575 \mathrm{~nm}$ long-pass filter before being routed toward two cross-polarized APDs. Varying power densities from $0.9 \mathrm{~kW} / \mathrm{cm}^{2}$ (Figure $\left.9(\mathrm{a})\right)$ up to $6.3 \mathrm{~kW} / \mathrm{cm}^{2}$ (Figure 9(i)) were used. In Figure 9, we display a series of images of the same region of the sample illuminated with increasing powers. Note that the molecular concentration used here is slightly higher than what is typically used for single-molecule fluorescence experiments so that several fluorophores may be excited at each position. From these images, we extracted the individual response of four dimer-molecule assemblies D1 to D4 and plotted as a function of incident power density (Figure 9). We clearly highlighted the linear behavior at low power (density $<2 \mathrm{~kW} / \mathrm{cm}^{2}$ ) mainly due to the molecular fluorescence. As the power increases, the dimer contribution is evolving going from linear to quadratic. In addition, at higher powers, the nanoantennas response becomes predominant with behavior from quadratic to supraquadratic varying from antennas to antennas. The broad distribution between dimers induced again a large distribution in the nonlinearity level $\mathrm{m}$ (with a main contribution for $m \approx 2$ over all dimers imaged here). This is to be expected, as the local field enhancement is particularly conducive to the enhancement of nonlinear processes such as two-photons absorption. Furthermore, enhancements are expected to be stronger under two photon excitation since its rate varies as the square of the local field intensity. Non-linear absorption processes also need a high-power density explaining our observation of a nonlinear response for high-power density. Thus, an excitation power sweep may serve to unravel molecular response from the nanoantennas in order to map the latter positions in densely coated samples. We now turn to measurement of the fluorescence lifetime in the low $\left(\sim 2 \mathrm{~kW} / \mathrm{cm}^{2}\right)$ excitation regime.

Figure 10 shows a fluorescence image of a sample with a concentration of perylene molecules slightly higher than concentration typically used for resolving fluorescence from single molecules. Many spots in the sample appear singlecolored indicating polarized fluorescence emission and thus the presence of only one or few molecules. Upon close inspection, a regular pattern of spots, reminiscent of the array of nanoantennas, appears, some of which are clearly brighter than the average spots. This brighter appearance may result from antenna-enhanced emission, locally higher concentrations of perylene (induced by the topography presented by the dimers during spin-coating), or a joint signal of molecular fluorescence and nanoantenna luminescence. In Figure 10, we present intensity time trajectories recorded at two positions that correlate with positions of dimer nanoantennas. These positions are indicated with numbered circles in the image. Both trajectories show blinking between different intensity levels in the first 10 to 20 seconds, together with discrete, permanent steps to a lower intensity, reminiscent of stepwise photobleaching of each of the perylenes. This blinking and stepwise bleaching is a clear indication of the presence of our perylene molecules [99]. From the number of discrete intensity levels, we can determine the number of molecules excited by the diffraction-limited laser focus. For instance, four separate intensity levels are identified in the first 20 seconds of 


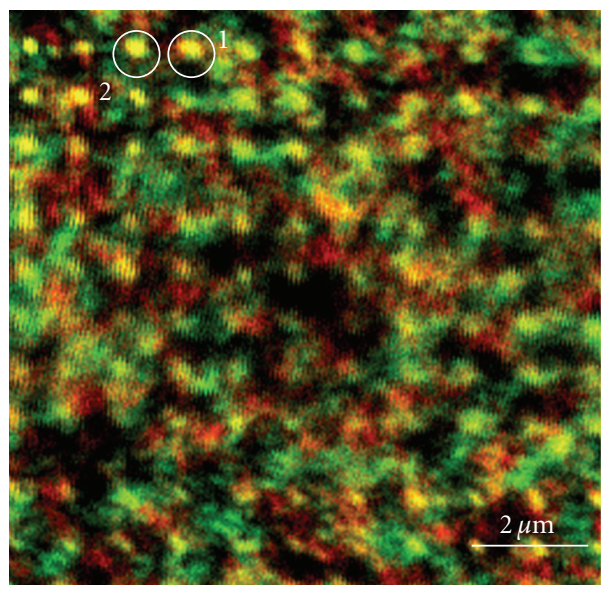

(a)

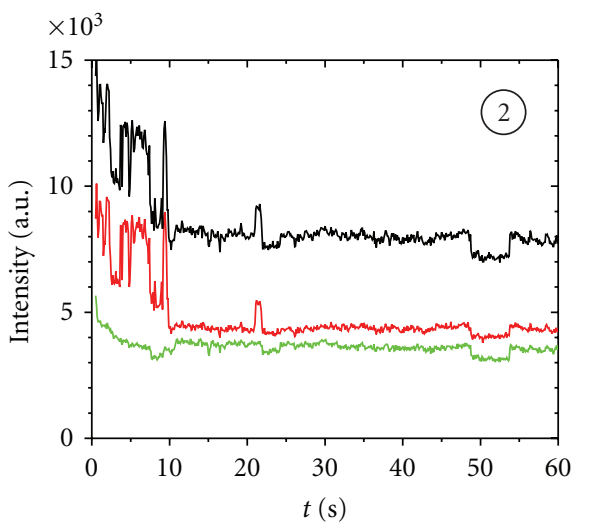

(d)

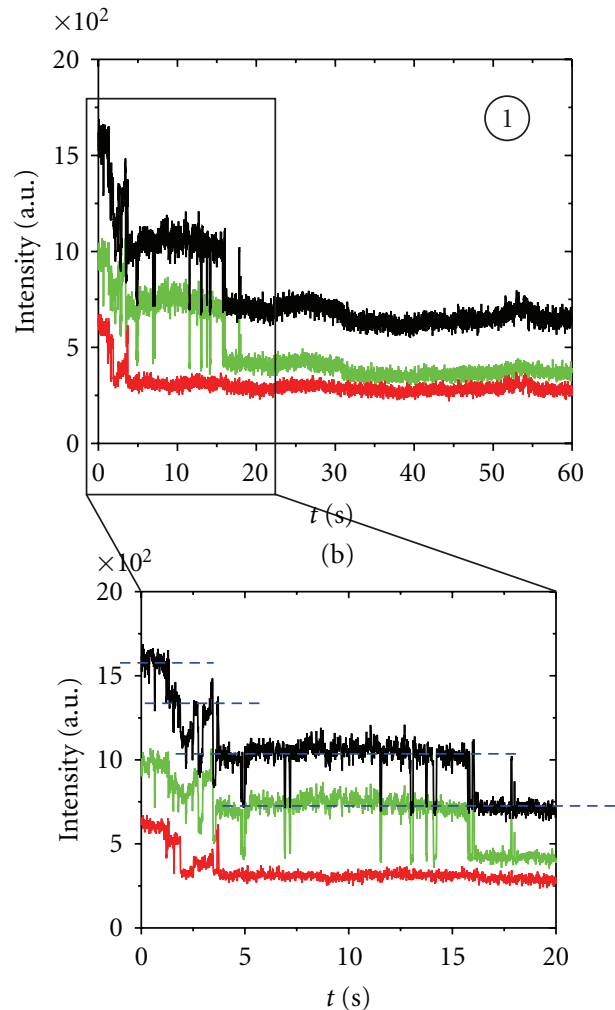

(c)

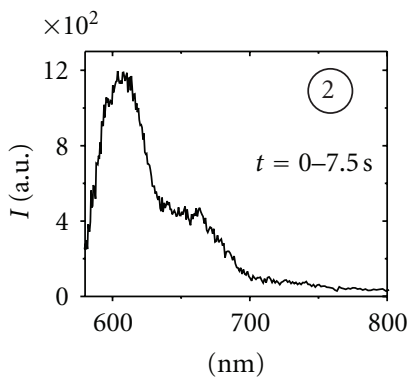

(e)

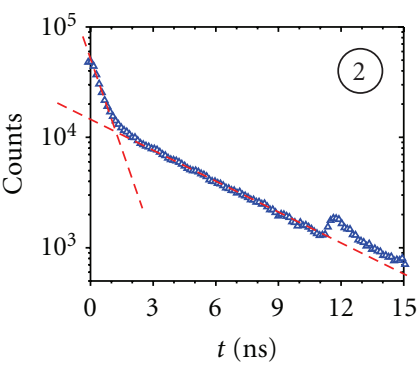

(f)

FIGURE 10: (a) Fluorescence image of perylene fluorescent molecules spin-coated on a substrate with dimer and monomer nanoantennas. (b) Fluorescence intensity trajectory measured at the position is indicated with circle numbered 1 in (a). Red and green trajectories indicate signals with orthogonal in-plane polarizations, while the black line indicates the total intensity. The intensity trajectory at position (1) shows a clear multilevel intensity blinking and bleaching reminiscent of molecular fluorescence. (c) Zoom in to the first $20 \mathrm{~s}$ where the four intensity levels are indicated with dashed blue lines. (d) The intensity trajectory measured at position number 2 in (a) displays similar blinking. (e) The fluorescence spectrum is recorded in the initial $7.5 \mathrm{~s}$ of the trajectory at position 2, with the spectra measured between $t=30$ and $t=60 \mathrm{~s}$ subtracted. The resulting spectrum is a typical emission spectrum of our perylene molecule. (f) Fluorescence lifetime is measured at position 2. Dashed lines in the lifetime graph indicate exponential fits with lifetimes of $\tau_{1}=0.4 \mathrm{~ns}$ and $\tau_{2}=5 \mathrm{~ns}$, respectively.

trajectory (1), as indicated in the figure. Of these, the three highest intensity levels show the discrete switching, while the fourth level remains continuous with broad variations on a 5-10 seconds timescale. We attribute the discrete switching behavior to the presence of three perylene molecules, while the fourth level constitutes a background signal that was present without blinking over observations as long as several minutes. Note also that the polarization anisotropy of the emitted fluorescence is different for the different intensity levels. Due to the fixed molecular orientation, and thus fixed transition dipole orientation, in the polymer matrix, this again indicates that the discrete intensity steps result from individual molecules switching on and off.

The trajectory recorded at position (2) displays a similar blinking before the signal falls to a continuous background. The small stepwise variations that are present in the background signal at $t \sim 20 \mathrm{~s}$ and $t \sim 50 \mathrm{~s}$ may result from molecules that are at the rim of the laser focus and are thus only weakly excited. In general, we observed similar trajectories with an initial discrete switching between typically one to three intensity levels and a remaining persistent continuous background signal for many different positions. All these 
positions coincided with the regular pattern of the nanoantennas array. Measurements at positions outside the antenna array yielded similar molecular fluorescence blinking but without this strong background signal. Thus, we attribute the persistent background signal to the presence of the dimer nanoantenna.

For all positions, fluorescence intensity spectrum and fluorescence lifetime were measured simultaneously with the intensity trajectory. For position (2) in Figure 10, we present the fluorescence spectrum recorded over the first 7.5 seconds of the trajectory. Here, the spectrum recorded for the background signal was subtracted, which yields a typical perylene emission spectrum. This again constitutes a strong indication that the initial intensity switching results from the presence of a perylene molecule. In the lower right panel of Figure 10, also the fluorescence decay curve (recorded from the photon arrival times after the laser trigger pulse) constructed from the 60-second trajectory is indicated. Here, we observe a clear biexponential decay with a fast component (measured lifetime $\tau_{1}=0.4 \mathrm{~ns}$, close to our instrument response and a slower component with $\tau_{2}=$ $5.0 \mathrm{~ns})$. The $5.0 \mathrm{~ns}$ lifetime is typical for our perylene dye in PMMA [98], while the fast component is attributed to the nanoantenna background signal. Similar decay curves with a fast component limited by our detector response and a slower component with typical perylene fluorescence lifetime were measured for all intensity trajectories that displayed the initial discrete fluorescence switching. From the fact that the measured fluorescence lifetimes are comparable to the regular (i.e., without nanoantennas) perylene lifetime, we conclude that these molecules are not coupled to the nanoantenna. The fast component of course may result from either antenna-coupled molecular emission or from the intrinsic luminescence of the nanoantennas. To resolve this latter issue, we reduced the molecular concentration to $\sim 10^{-8} \mathrm{M}$, a typical concentration for single-molecule fluorescence experiments. In this case, the dye dissolved in toluene was first spin-coated (i.e., without PMMA), while a PMMA solution in toluene was spin-coated afterwards to coat with a protective $\sim 50 \mathrm{~nm}$ thick layer of polymer. With this procedure, we expect topographic effects, that is, capillary interactions, during spin-coating of the perylene solution to draw the molecules to the nanoantennas. Given the overall low concentration of perylene molecules, even in this case we expect only a few nanoantennas to be coupled with a molecule. In Figure 11, a typical fluorescence image from an area with nanoantennas is shown.

Fluorescence spectra were recorded at different nanoantenna positions as indicated in Figure 11. All spectra show a resonance below $620 \mathrm{~nm}$ (overlapping with the molecular excitation and emission wavelength as anticipated) and extend into the red part of the wavelength spectrum. All nanoantenna spectra show a dip at $630 \mathrm{~nm}$. We grouped our measured dimer antenna spectra in two groups based on the appearance of the fluorescence spectra beyond this $630 \mathrm{~nm}$ dip. The group indicated with red curves extends deep into the $700 \mathrm{~nm}$ range with three broad peaks at equivalent positions between $630 \mathrm{~nm}$ and $700 \mathrm{~nm}$. Compared to the molecular fluorescence spectra also indicated in Figure 11,

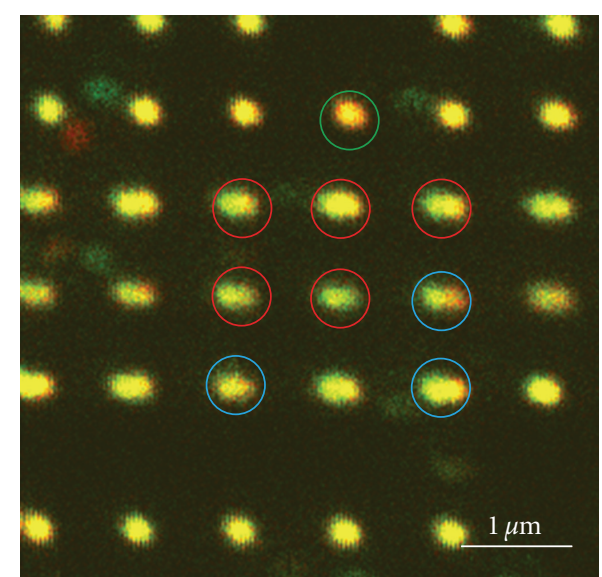

(a)

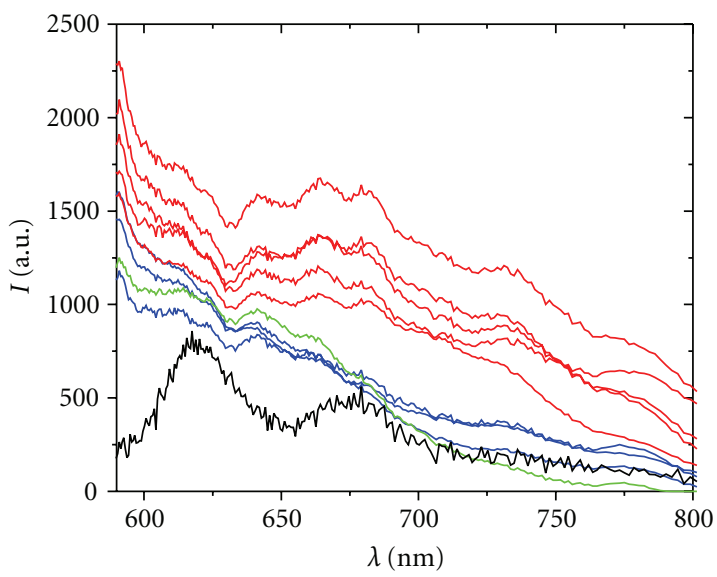

(b)

FIgURE 11: (a) Fluorescence image of a nanoantenna array with low concentration of perylene molecules. Bright spots indicate the positions of nanoantennas. First two rows and the lowest row are monomer antennas, while the middle three rows are dimer antennas. (b) Fluorescence spectra for eight dimer positions and one monomer position from the image in (a). The spectra with red lines correspond to red-encircled spots in the image, blue curves to the blue-encircled spots, and the green curve to the monomer position. The black curve indicates a typical perylene fluorescence spectrum recorded for a single molecule outside the nanoantenna array.

we can see that the nanoantenna response shows a good overlap with the molecular resonance. The curves indicated in blue show a faster decay below $700 \mathrm{~nm}$ and are reminiscent of the monomer spectra indicated with the green curve. Thus, we attribute these latter to geometrical artifacts (such as fused dimers) and assume the red curves to result from proper gap dimers. For all these positions, fluorescence lifetime was measured simultaneously, and this always resulted in a fast, $\tau \sim 0.5 \mathrm{~ns}$ decay without any sign of slower component resulting from uncoupled molecules. In addition, the intensity trajectories were similar to the background signal presented in the respective panels in Figure 10. Similar behavior was observed for several tens 


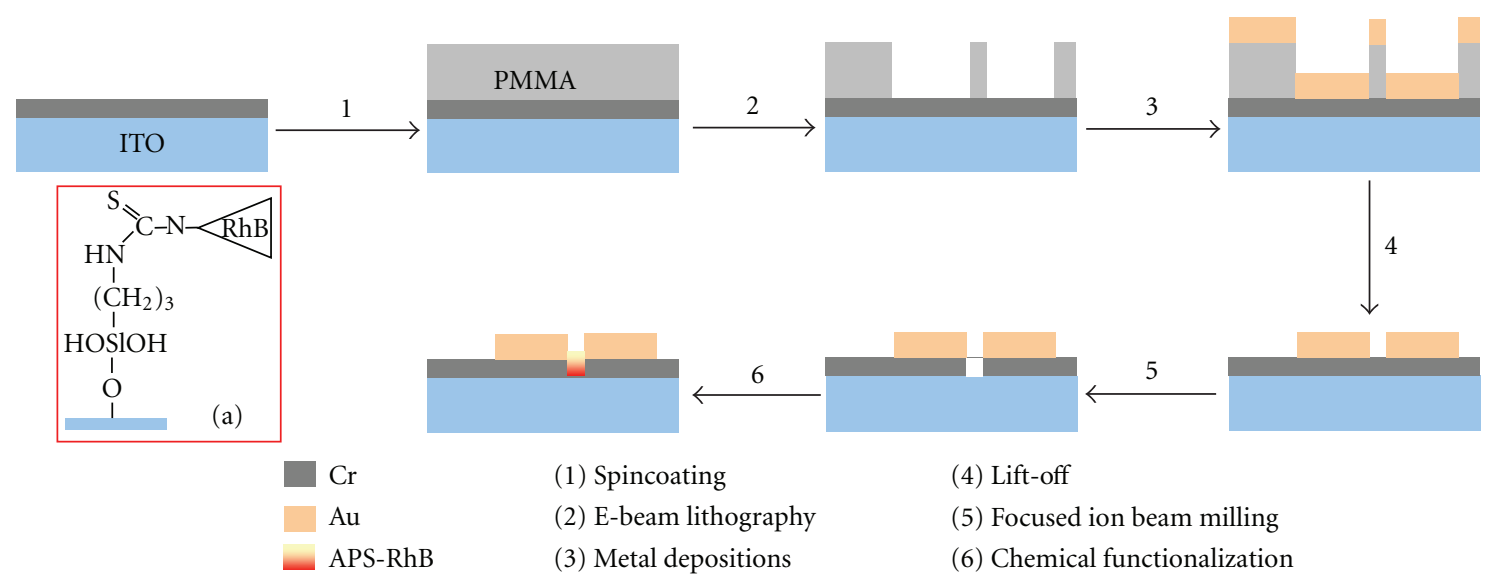

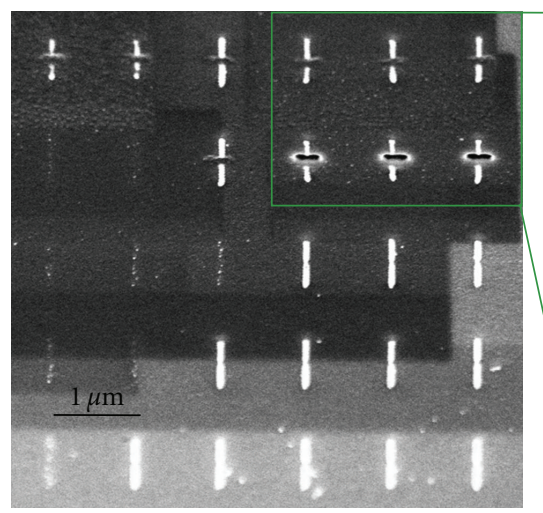

(b)

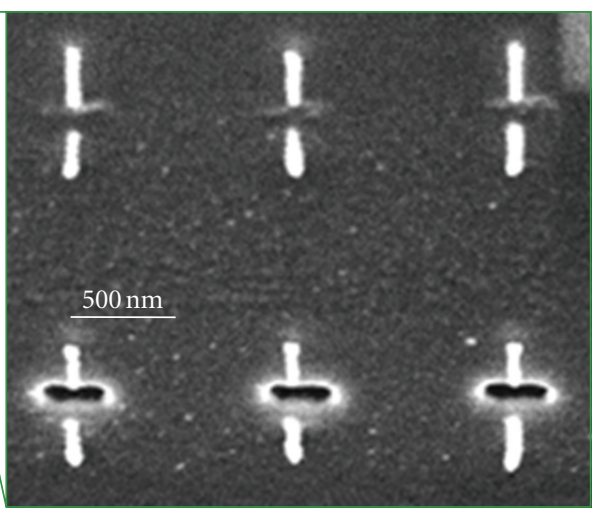

(c)

FIGURE 12: Schematic outline of the fabrication procedure of a molecular plasmonic nanoantenna involving the consecutive application of electron-beam lithography, focused ion beam (FIB) milling, and localized chemical surface functionalization. The inset (a) displays the surface grafting of a Rhodamine B (RhB) fluorophore onto the ITO substrate. The FIB step (step 5) is represented by the SEM images in (b) and (c) where the gap is milled either through a few nanometers of chromium only (top row in (c)) or all the way to the ITO layer (bottom row in (c)).

of nanoantenna positions, where statistically a majority of these are expected not to be coupled to molecules. While few of the recorded fluorescence spectra could result from a strongly coupled antenna-molecule system, such fluorescence measurements fail to discriminate between the signal of a coupled system and the intrinsic luminescence of the dimer nanoantennas. Note that the comparatively higher luminescence signal for this system compared to the previous DiD system, may result from the shorter pulse length, and thus higher instantaneous power density, in the perylene experiments ( $280 \mathrm{fs}$ as compared to $90 \mathrm{ps}$ ). Finally, we want to note that in this strong coupling regime, molecules coupled to an antenna would predominantly radiate via the antenna modes. Thus, molecular positions can only be retrieved in a weaker coupling regime. In this case, however, the fact that both molecular lifetime distribution and nanoantenna field strength distribution (see Section 3) have to be statistically accounted for makes that hundreds of positions need to be measured which is a laborious task. Thus, we can conclude that only an a priori positioning of fluorescent molecules at well-defined positions with respect to the nanoantenna can solve these issues. In the next section, we will present a fabrication scheme with which this could be achieved.

\section{Dedicated Positioning of Molecules in the Nanoantenna Gap}

To tackle the issues presented above, we devised a fabrication procedure for molecular plasmonic nanostructures where functional molecules can be placed at a predefined position which is the gap of the nanoantennas. Luminescence measurements were thereafter carried out as a proof of principle.

Our methodology relies on the use of two distinct conductive layers which allows the consecutive, high-resolution application of different charged particle lithography techniques, in our case electron-beam lithography and focused ion beam milling. The top conductive layer furthermore serves both as a deposition and adhesion layer for the plasmonic structure and as a mask for a final chemical surface modification step, which functionalizes the exposed parts of the underlying conductive material. An outline of the fabrication procedure is given in Figure 12. First, we 


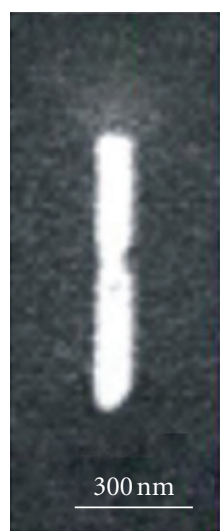

(a)

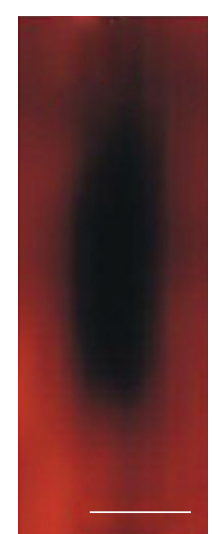

(b)

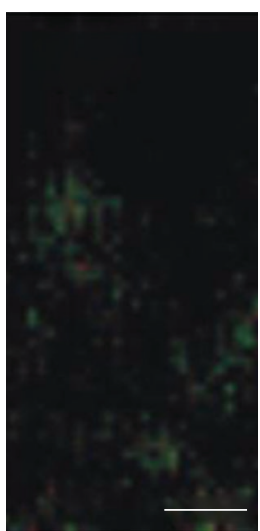

(c)

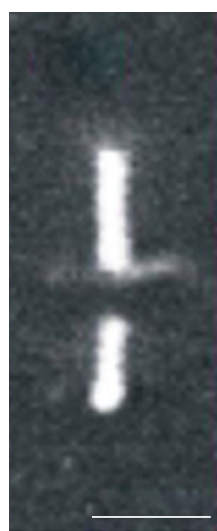

(d)

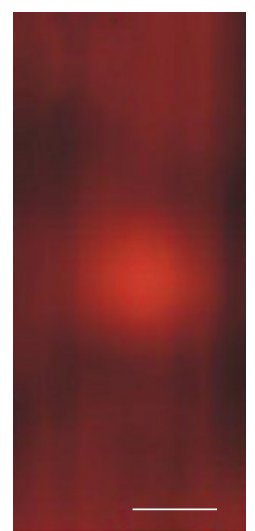

(e)

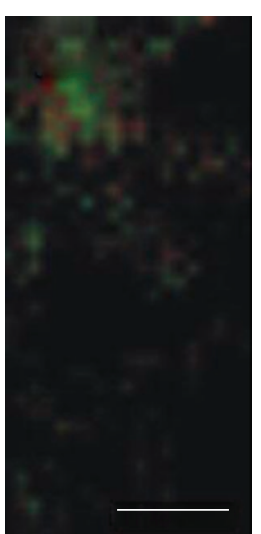

(f)

FIGURE 13: (a-c) Gold nanoantenna not exposed to FIB milling after surface functionalization: (a) SEM image, (b) optical transmission microscopy image, and (c) fluorescence microscopy image. (d-f) Same respective images for a gold nanoantenna where the gap size had been increased by FIB milling but without exposing the lower ITO layer.

evaporate a $80 \mathrm{~nm}$ thick layer of indium tin oxide (ITO) onto a glass cover slide. The ITO layer is overcoated with an approximately $10 \mathrm{~nm}$ layer of chromium by evaporation. The use of optically transparent ITO allows for light microscopy in both reflection and transmission mode and thus makes the fabrication scheme compatible for applications relying on enhanced detection of, for example, fluorescence or Raman scattering signals. Gold nanoantennas were created on the $\mathrm{Cr}$ layer with the process described in Section 2 (Figure 1), the $\mathrm{Cr}$ layer replacing the $\mathrm{Ti}$ adhesion layer. The nanoantennas consist here of two equally sized gold rods $(l \times w \times h=$ $300 \times 80 \times 40 \mathrm{~nm}$ ) with the short ends facing at a separation of $30 \mathrm{~nm}$. Examples of such gold nanoantennas are given in the bottom row of Figure 12(b) or closely in Figure 13(a).

Next, we use focused ion beam (FIB) lithography to mill a rectangular, $200 \mathrm{~nm}$ long, $50 \mathrm{~nm}$ wide hole into the $\mathrm{Cr}$ layer, cutting the nanoantennas halfway, that is, at the gap. This increases the gap size of exposed nanoantennas to approximately $50 \mathrm{~nm}$ (see Figure 12(c)). The FIB milling finally uncovers the underlying ITO substrate (step 5 in Figure 12). These exposed ITO areas on the substrate can be selectively functionalized using a surface grafting procedure that exploits the chemical contrast between metal $(\mathrm{Cr})$ and oxide (ITO) areas. If necessary the Cr surface can be passivated by applying a coating reaction prior to FIB milling. We functionalized the exposed ITO area in the gap between both gold bars with a Rhodamine dye derivative. Rhodamine dyes are often used both as fluorescent markers because of their high quantum yield and photostability and as model probes for surface-enhanced Raman scattering studies. First, isothiocyanate Rhodamine $\mathrm{B}(\mathrm{RhB})$ was linked to the silane coupling agent 3-aminopropyltriethoxysilane (APS). The ITO areas are then reacted to the APS-RhB construct (see Figure 12(a)) under ammonia-catalyzed conditions [100]. Samples were thoroughly rinsed with ethanol to prevent absorption of APS-RhB onto the $\mathrm{Cr}$ surface upon drying.
The resulting nanostructure is indicated in Figure 12 after step 6.

After APS-RhB reaction, gold nanoantennas were analyzed using the previously described high-resolution (singlemolecule sensitivity) confocal microscopy setup in both transmission (excitation light) and reflection (fluorescence light) modes. Nanoantennas not exposed to FIB milling are visible due to decreased transmission, but do not show fluorescence (Figures 13(b) and 13(c)). Milling a gap in the nanoantenna and a small indentation in the Cr-layer without exposing the ITO substrate (Figures 13(d), 13(e), and 13(f)) leads to a corresponding small increase in transmission, at the location of the indentation in between the gold rods. However, no change in fluorescence signal from background level is observed, indicating negligible absorption of APS$\mathrm{RhB}$ onto the Cr surface. We have used a range of FIB milling times and beam doses, which leads to various indentation depths in the Cr layer, but a fluorescence signal was only observed when the transmission signal indicated removal of the Cr layer. At this point, transmission increased about 6fold compared to transmission through the non-FIB-milled Cr areas.

Figure 14 shows results for nanoantennas where FIB milling completely removed the $\mathrm{Cr}$ layer, leading to local exposure of ITO. Removal of the Cr layer is evidenced by the increase in the transmission signal, and RhBfunctionalization of the ITO in the gap gives rise to a strong fluorescence signal. The width of the Gaussian fluorescence profiles is $310 \mathrm{~nm}$, that is, diffraction limited. This is in correspondence with the projected gap size of $200 \times 50 \mathrm{~nm}$. Note that the alignment of the FIB area with nanoantenna gap is not optimal so that antennas are partly cut. The differences in fluorescence intensity between the functionalized nanoantennas can be caused by variations in the number of $\mathrm{RhB}$ molecules. In principle, this could also be caused by the field enhancement variations but given the relatively large gap sizes and the fact that antennas are 


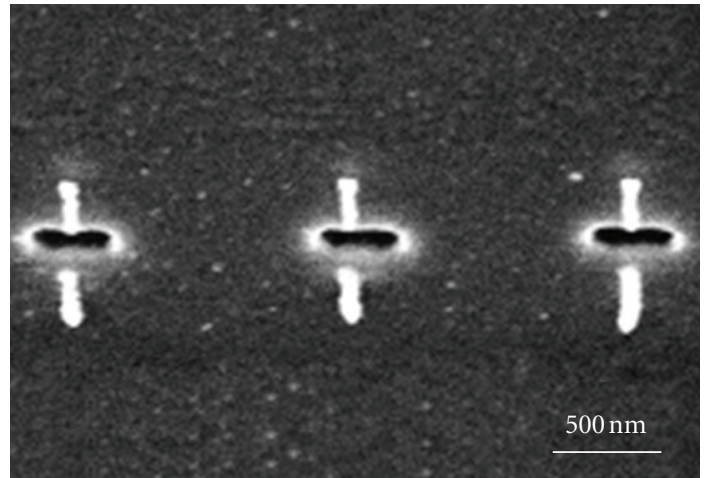

(a)

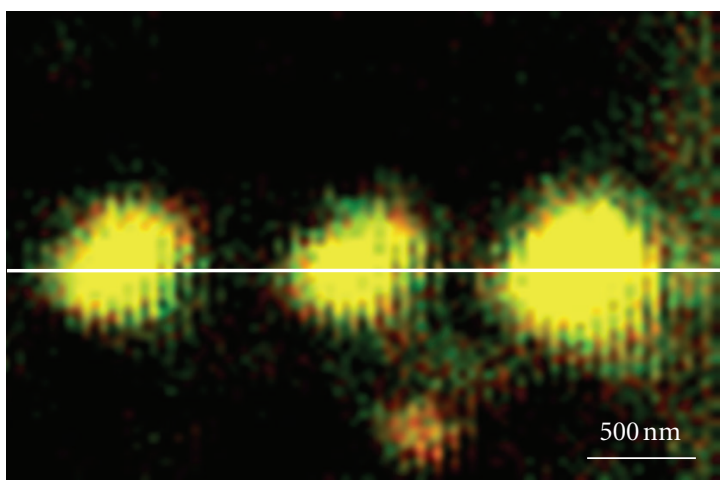

(c)

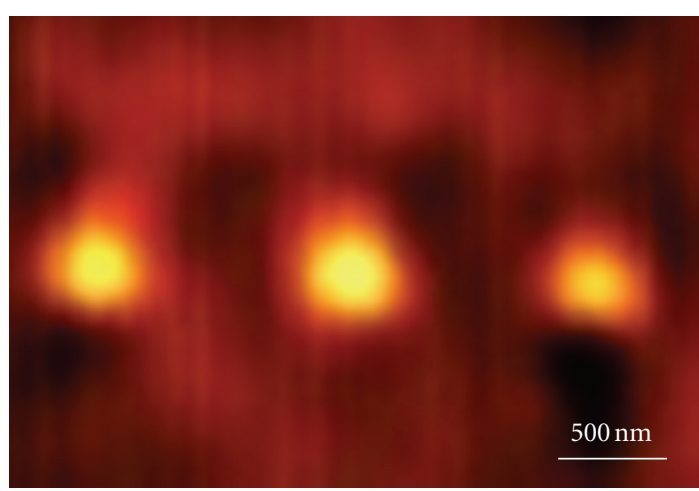

(b)

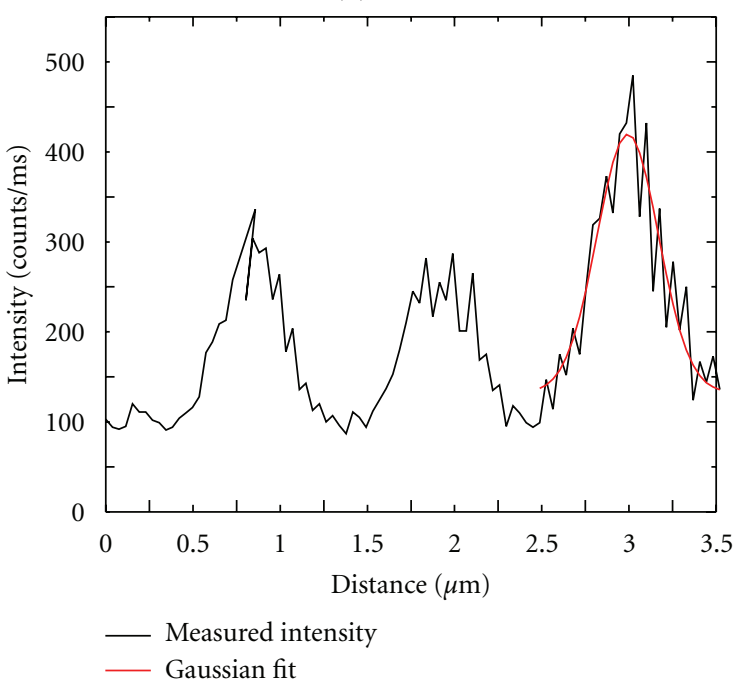

(d)

FIgURE 14: (a) SEM image of gold nanoantennas after FIB milling through the Cr layer and the respective images in (b) transmission and (c) fluorescence microscopy. Color scaling in (b, c) is identical to that in Figure 2 to highlight the intensity increase. The fluorescence image has been built from a red and a green channel-denoting orthogonal polarization directions. (d) Intensity profile along the white line drawn in (c) indicating that fluorescence spots are diffraction limited.

partly cut by the FIB procedure, we do not expect significant enhancement. This is also indicated by the unpolarized and thus not-antenna-coupled emission. By adjusting reagent concentrations, surface coating could be tuned to a few or single molecules per nanostructure, which would allow for a detailed and localized mapping of field enhancement properties around plasmonic nanostructures.

In summary, we have presented a fabrication procedure for the localized, sub-diffraction-limit positioning of fluorescent molecules at a designed position with respect to a plasmonic nanoantenna. Initial results indicate the feasibility of the approach, which relies on the combined use of electron-beam and focused ion beam lithography, together with chemical surface modification. The use of two distinct conductive layers, of which the upper one serves as a mask for the chemical functionalization, allows both lithography techniques to be applied at high resolution, opening the way to sub-20 nm patterning. Further work should aim at demonstrating the technique at this resolution and at improving alignment with the nanoantennas. This would give the possibility to unambiguously map the local field around plasmonic nanostructures, but also provide the way for creating active molecular plasmonic nanodevices for, for example, high-resolution chemical and biological sensing, light harvesting, and nanophotonic components.

\section{Conclusions and Perspective}

Gap nanoantennas composed of double identical rods separated by a nanometric gap were first fabricated by electron beam lithography before being theoretically investigated and experimentally characterized by far-field spectroscopy. In a preliminary study, arrays of gap nanoantennas with various aspect ratios and gap sizes were considered and their optical response recorded. The local field in the close vicinity of the nanoantennas was then evaluated by luminescent measurements using a confocal microscope with single-molecule detection capability. Polarization effects were then explored. We observed luminescence predominantly polarized along 
the dimer axis with an average enhancement up to 3-fold. This is in agreement with previous work reporting 10-fold enhancement on similar arrays using two-photon luminescence measurements. These prior studies give insights into the local response distribution of such metallic nanoantennas and constituted a strong guiding line when tackling the issue of nanoantenna-single molecule coupling.

It is crucial to understand antenna-molecule coupling as these hybrid systems receive increasing attention in fields as diverse as biosensing, imaging, or photovoltaics. As a general strategy, we used single fluorescent molecules as vectorial nonperturbative probes to map the local field of individual nanoantennas. For this task, two types of fluorophores were used. First, common DiD fluorophores were randomly deposited over the nanoantenna arrays. We observed enhancement of the fluorescence intensity caused by the presence of nanoantennas. However, and despite the low excitation power used in the experiment, it was difficult to dissociate the molecular fluorescence from the intrinsic gold luminescence signal. In addition, the presence of the nanoantennas could disturb the homogeneity of the fluorophores on the samples, precluding quantitative analysis. To discriminate between the fluorophore and the nanoantenna contributions to the fluorescence signal, we subsequently utilized perylene molecules as photostable fluorophores. They permitted longer observations (up to minutes). In addition, they exhibit an on-off switching on a timescale of seconds. These combined features eased the discrimination between the molecular and nanoantenna contribution to the fluorescence signal. We then carried out fluorescence measurements at different excitation power. Due to the different power laws followed by linear molecular fluorescence and two-photon excited luminescence from the nanoantennas, we were able to discriminate these two contributions. Fluorescence spectroscopy and lifetime measurements were then carried out on the nanoantennamolecule system. The lifetime measurements revealed a biexponential decay with a fast component $\tau_{1}=0.4 \mathrm{~ns}$ (close to the system temporal resolution) attributed to the nanoantennas background and a typical $\tau_{2}=5.0 \mathrm{~ns}$ typical of perylene in PMMA. From the insensitivity of the latter decay to the presence of the nanoantennas, we conclude that the molecules under investigation were not coupled to nanoantennas. By cons, the fast component is linked to the nanoantennas whether via antenna-coupled molecular emission or as a result of the intrinsic antenna luminescence. Even working at low concentration, and therefore being less influenced by the topographic artifacts, we expected to have only rare antenna-molecule coupling events. Furthermore, we note that any molecule coupled to an antenna should reemit via the antenna modes, and therefore a large number of positions have to be evaluated to take into account the broad distributions in the molecules' lifetime and in the dimers' luminescence. To overcome this issue, we proposed a hybrid system composed of a nanoantenna and a molecule with well-controlled position. Its feasibility was demonstrated using a process involving electron beam and focused ion beam lithography, followed by chemical surface modification. This opens up new routes toward the local 3D mapping of local field in the vicinity of metallic nanoantennas as well as active plasmonic nanodevices, where the relative positioning remains a burden. This has tremendous applications in the numerous currently hot topics such as the development of nanophotonic components, bio- and chemical sensing, imaging, light-harvesting mimicking systems, or hybrid photovoltaic cells design.

\section{Acknowledgment}

The authors would like to thank: Professor Ali Passian at the Oak Ridge National Laboratory in Tennessee for providing the theoretical results illustrated in Figures 2 and 3 of Section 2; Frans Segerink at University of Twente and M. J. Lopez Bosque at the Parc Científic de Barcelona for FIB-milling; Petru Ghenuche and Gabriel Sanchez-Mosteiro at ICFO-Barcelona for e-beam lithography and confocal microscopy, and A. van Blaaderen for use of laboratory facilities. This work was supported by the EU Network of Excellence Plasmo Nano Devices (WP 19.8.2). Niek van Hulst acknowledges funding by the European Specific Target Research Project (STRP) ASPRINT, the Spanish MICINN program CONSOLIDER CSD2007-046, Fundacio CELLEX Barcelona and the EU ERC Grant No. 247330. Aude L. Lereu acknowledges the Centre National de la Recherche Scientifique.

\section{References}

[1] P. Bharadwaj, B. Deutsch, and L. Novotny, "Optical antennas," Advances in Optics and Photonics, vol. 1, pp. 438-483, 2009.

[2] L. Novotny and N. F. van Hulst, "Antennas for light," Nature Photonics, vol. 5, pp. 83-90, 2011.

[3] C. Girard and E. Dujardin, "Near-field optical properties of top-down and bottom-up nanostructures," Journal of Optics A, vol. 8, supplement 4, pp. S73-S86, 2006.

[4] F. Wang and Y. R. Shen, "General properties of local plasmons in metal nanostructures," Physical Review Letters, vol. 97, no. 20, Article ID 206806, 2006.

[5] C. Girard, "Near fields in nanostructures," Reports on Progress in Physics, vol. 68, no. 8, pp. 1883-1933, 2005.

[6] S. A. Maier and H. A. Atwater, "Plasmonics: localization and guiding of electromagnetic energy in metal/dielectric structures," Journal of Applied Physics, vol. 98, no. 1, Article ID 011101, pp. 1-10, 2005.

[7] H. Cang, A. Labno, C. Lu et al., "Probing the electromagnetic field of a 15-nanometre hotspot by single molecule imaging," Nature, vol. 469, no. 7330, pp. 385-388, 2011.

[8] P. Ghenuche, R. Quidant, and G. Badenes, "Cumulative plasmon field enhancement in finite metal particle chains," Optics Letters, vol. 30, no. 14, pp. 1882-1884, 2005.

[9] K. Imura, T. Nagahara, and H. Okamoto, "Near-field two-photon-induced photoluminescence from single gold nanorods and imaging of plasmon modes," Journal of Physical Chemistry B, vol. 109, no. 27, pp. 13214-13220, 2005.

[10] K. Imura, T. Nagahara, and H. Okamoto, "Photoluminescence from gold nanoplates induced by near-field twophoton absorption," Applied Physics Letters, vol. 88, no. 2, Article ID 023104, pp. 1-3, 2006. 
[11] P. Mühlschlegel, H. J. Eisler, O. J. F. Martin, B. Hecht, and D. W. Pohl, "Applied physics: resonant optical antennas," Science, vol. 308, no. 5728, pp. 1607-1609, 2005.

[12] P. J. Schuck, D. P. Fromm, A. Sundaramurthy, G. S. Kino, and W. E. Moerner, "Improving the mismatch between light and nanoscale objects with gold bowtie nanoantennas," Physical Review Letters, vol. 94, no. 1, Article ID 017402, 2005.

[13] A. Sundaramurthy, P. J. Schuck, N. R. Conley, D. P. Fromm, G. S. Kino, and W. E. Moerner, "Toward nanometer-scale optical photolithography: utilizing the near-field of bowtie optical nanoantennas," Nano Letters, vol. 6, no. 3, pp. 355360, 2006.

[14] A. K. Sheridan, A. W. Clark, A. Glidle, J. M. Cooper, and D. R. S. Cumming, "Multiple plasmon resonances from gold nanostructures," Applied Physics Letters, vol. 90, no. 14, Article ID 143105, 2007.

[15] C. E. Talley, J. B. Jackson, C. Oubre et al., "Surface-enhanced Raman scattering from individual Au nanoparticles and nanoparticle dimer substrates," Nano Letters, vol. 5, no. 8, pp. 1569-1574, 2005.

[16] A. M. Michaels, J. Jiang, and L. Brus, "Ag nanocrystal junctions as the site for surface-enhanced raman scattering of single rhodamine 6G molecules," Journal of Physical Chemistry B, vol. 104, no. 50, pp. 11965-11971, 2000.

[17] K. Li, M. I. Stockman, and D. J. Bergman, "Self-similar chain of metal nanospheres as an efficient nanolens," Physical Review Letters, vol. 91, no. 22, Article ID 227402, 4 pages, 2003.

[18] L. Novotny and B. Hecht, Principles of Nano-Optics, Cambridge University Press, 2006.

[19] L. Novotny and S. J. Stranick, "Near-field optical microscopy and spectroscopy with pointed probes," Annual Review of Physical Chemistry, vol. 57, pp. 303-331, 2006.

[20] L. Novotny, "Effective wavelength scaling for optical antennas," Physical Review Letters, vol. 98, no. 26, Article ID 266802, 2007.

[21] A. Hartschuh, M. R. Beversluis, A. Bouhelier, and L. Novotny, "Tip-enhanced optical spectroscopy," Philosophical Transactions of the Royal Society A, vol. 362, no. 1817, pp. 807-819, 2004.

[22] A. Hartschuh, "Tip-enhanced near-field optical microscopy," Angewandte Chemie, vol. 47, no. 43, pp. 8178-8191, 2008.

[23] E. Bailo and V. Deckert, "Tip-enhanced Raman scattering," Chemical Society Reviews, vol. 37, no. 5, pp. 921-930, 2008.

[24] E. Fort and S. Grésillon, "Surface enhanced fluorescence," Journal of Physics D, vol. 41, no. 1, Article ID 013001, 2008.

[25] A. L. Lereu, A. Passian, and P. Dumas, "Near field optical microscopy: a brief review," International Journal of Nanotechnology, vol. 8, 2011.

[26] S. Wedge, J. A. E. Wasey, W. L. Barnes, and I. Sage, "Coupled surface plasmon-polariton mediated photoluminescence from a top-emitting organic light-emitting structure," Applied Physics Letters, vol. 85, no. 2, pp. 182-184, 2004.

[27] C. Liu, V. Kamaev, and Z. V. Vardeny, "Efficiency enhancement of an organic light-emitting diode with a cathode forming two-dimensional periodic hole array," Applied Physics Letters, vol. 86, no. 14, Article ID 143501, pp. 1-3, 2005.

[28] E. Ozbay, "Plasmonics: merging photonics and electronics at nanoscale dimensions," Science, vol. 311, no. 5758, pp. 189 193, 2006

[29] S. Pillai, K. R. Catchpole, T. Trupke, G. Zhang, J. Zhao, and M. A. Green, "Enhanced emission from Si-based lightemitting diodes using surface plasmons," Applied Physics Letters, vol. 88, no. 16, Article ID 161102, 2006.
[30] G. Sun, J. B. Khurgin, and R. A. Soref, "Plasmonie lightemission enhancement with isolated metal nanoparticles and their coupled arrays," Journal of the Optical Society of America $B$, vol. 25, no. 10, pp. 1748-1755, 2008.

[31] P. Ball, "Let there be light," Nature, vol. 409, no. 6823, pp. 974-976, 2001.

[32] R. Corkish, M. A. Green, and T. Puzzer, "Solar energy collection by antennas," Solar Energy, vol. 73, no. 6, pp. 395401, 2002.

[33] P. J. Burke, S. Li, and Z. Yu, "Quantitative theory of nanowire and nanotube antenna performance," IEEE Transactions on Nanotechnology, vol. 5, no. 4, Article ID 1652847, pp. 314334, 2006.

[34] K. Kempa, J. Rybczynski, Z. Huang et al., "Carbon nanotubes as optical antennae," Advanced Materials, vol. 19, no. 3, pp. 421-426, 2007.

[35] S. Pillai, K. R. Catchpole, T. Trupke, and M. A. Green, "Surface plasmon enhanced silicon solar cells," Journal of Applied Physics, vol. 101, no. 9, Article ID 093105, 2007.

[36] K. Nakayama, K. Tanabe, and H. A. Atwater, "Plasmonic nanoparticle enhanced light absorption in GaAs solar cells," Applied Physics Letters, vol. 93, no. 12, Article ID 121904, 2008.

[37] S. H. Lim, W. Mar, P. Matheu, D. Derkacs, and E. T. Yu, "Photocurrent spectroscopy of optical absorption enhancement in silicon photodiodes via scattering from surface plasmon polaritons in gold nanoparticles," Journal of Applied Physics, vol. 101, no. 10, Article ID 104309, 2007.

[38] C. Hägglund, M. Zäch, G. Petersson, and B. Kasemo, "Electromagnetic coupling of light into a silicon solar cell by nanodisk plasmons," Applied Physics Letters, vol. 92, no. 5, Article ID 053110, 2008.

[39] A. J. Morfa, K. L. Rowlen, T. H. Reilly II, M. J. Romero, and J. Van De Lagemaat, "Plasmon-enhanced solar energy conversion in organic bulk heterojunction photovoltaics," Applied Physics Letters, vol. 92, no. 1, Article ID 013504, 2008.

[40] S. S. Kim, S. I. Na, J. Jo, D. Y. Kim, and Y. C. Nah, "Plasmon enhanced performance of organic solar cells using electrodeposited Ag nanoparticles," Applied Physics Letters, vol. 93, no. 7, Article ID 073307, 2008.

[41] D. Derkacs, W. V. Chen, P. M. Matheu, S. H. Lim, P. K. L. Yu, and E. T. Yu, "Nanoparticle-induced light scattering for improved performance of quantum-well solar cells," Applied Physics Letters, vol. 93, no. 9, Article ID 091107, 2008.

[42] A. Passian, S. Zahrai, A. L. Lereu, R. H. Farahi, T. L. Ferrell, and T. Thundat, "Nonradiative surface plasmon assisted microscale Marangoni forces," Physical Review E, vol. 73, no. 6, Article ID 066311, 2006.

[43] R. H. Farahi, A. Passian, S. Zahrai, A. L. Lereu, T. L. Ferrell, and T. Thundat, "Microscale Marangoni actuation: all-optical and all-electrical methods," Ultramicroscopy, vol. 106, no. 8-9, pp. 815-821, 2006.

[44] V. M. Shalaev, W. Cai, U. K. Chettiar et al., "Negative index of refraction in optical metamaterials," Optics Letters, vol. 30, no. 24, pp. 3356-3358, 2005.

[45] V. M. Shalaev, "Optical negative-index metamaterials," Nature Photonics, vol. 1, no. 1, pp. 41-48, 2007.

[46] G. W. Bryant, I. Romero, F. J. Garcia de Abajo, and J. Aizpurua, "Stimulating electromagnetic response in coupled metallic nanoparticles for nanoscale optical microscopy and spectroscopy: nanorod-end effects," in Plasmonics: Metallic Nanostructures and their Optical Properties, vol. 6323 of Proceedings of SPIE, pp. 632313-632318, 2006. 
[47] I. Romero, J. Aizpurua, G. W. Bryant, and F. J. García De Abajo, "Plasmons in nearly touching metallic nanoparticles: singular response in the limit of touching dimers," Optics Express, vol. 14, no. 21, pp. 9988-9999, 2006.

[48] J. Aizpurua, G. W. Bryant, L. J. Richter, F. J. García De Abajo, B. K. Kelley, and T. Mallouk, "Optical properties of coupled metallic nanorods for field-enhanced spectroscopy," Physical Review B, vol. 71, no. 23, Article ID 235420, 13 pages, 2005.

[49] I. D. Mayergoyz, Z. Zhang, and G. Miano, "Analysis of dynamics of excitation and dephasing of plasmon resonance modes in nanoparticles," Physical Review Letters, vol. 98, no. 14, Article ID 147401, 2007.

[50] C. Dahmen, B. Schmidt, and G. Von Plessen, "Radiation damping in metal nanoparticle Pairs," Nano Letters, vol. 7, no. 2, pp. 318-322, 2007.

[51] S. Foteinopoulou, J. P. Vigneron, and C. Vandenbem, “Optical near-field excitations on plasmonic nanoparticle-based structures," Optics Express, vol. 15, no. 7, pp. 4253-4267, 2007.

[52] I. Olivares, R. Rojas, and F. Claro, "Surface modes of a pair of unequal spheres," Physical Review B, vol. 35, no. 5, pp. 24532455, 1987.

[53] L. Gou and C. J. Murphy, "Fine-tuning the shape of gold nanorods," Chemistry of Materials, vol. 17, no. 14, pp. 36683672, 2005.

[54] M. Futamata, Y. Maruyama, and M. Ishikawa, "Local electric field and scattering cross section of Ag nanoparticles under surface plasmon resonance by finite difference time domain method," Journal of Physical Chemistry B, vol. 107, no. 31, pp. 7607-7617, 2003.

[55] S. Enoch, R. Quidant, and G. Badenes, "Optical sensing based on plasmon coupling in nanoparticle arrays," Optics Express, vol. 12, no. 15, pp. 3422-3427, 2004.

[56] J. P. Kottmann and O. J. F. Martin, "Plasmon resonant coupling in metallic nanowires," Optics Express, vol. 8, no. 12, pp. 655-663, 2001.

[57] H. Tamaru, H. Kuwata, H. T. Miyazaki, and K. Miyano, "Resonant light scattering from individual Ag nanoparticles and particle pairs," Applied Physics Letters, vol. 80, no. 10, p. 1826, 2002.

[58] A. Bouhelier, R. Bachelot, G. Lerondel, S. Kostcheev, P. Royer, and G. P. Wiederrecht, "Surface plasmon characteristics of tunable photoluminescence in single gold nanorods," Physical Review Letters, vol. 95, no. 26, Article ID 267405, 2005.

[59] H. Kuwata, H. Tamaru, K. Esumi, and K. Miyano, "Resonant light scattering from metal nanoparticles: practical analysis beyond rayleigh approximation," Applied Physics Letters, vol. 83, no. 22, pp. 4625-4627, 2003.

[60] A. A. Mikhailovsky, M. A. Petruska, M. I. Stockman, and V. I. Klimov, "Broadband near-field interference spectroscopy of metal nanoparticles using a femtosecond white-light continuum," Optics Letters, vol. 28, no. 18, pp. 1686-1688, 2003.

[61] D. Ten Bloemendal, P. Ghenuche, R. Quidant, I. G. Cormack, P. Loza-Alvarez, and G. Badenes, "Local field spectroscopy of metal dimers by TPL microscopy," Plasmonics, vol. 1, no. 1, pp. 41-44, 2006.

[62] P. Ghenuche, S. Cherukulappurath, T. H. Taminiau, N. F. Van Hulst, and R. Quidant, "Spectroscopic mode mapping of resonant plasmon nanoantennas," Physical Review Letters, vol. 101, no. 11, Article ID 116805, 2008.

[63] A. L. Lereu, G. Sanchez-Mosteiro, P. Ghenuche, R. Quidant, and N. F. Van Hulst, "Individual gold dimers investigated by far- and near-field imaging," Journal of Microscopy, vol. 229, no. 2, pp. 254-258, 2008.

[64] A. L. Lereu, G. Sanchez-Mosteiro, P. Ghenuche et al., "Probing the local field of nanoantennas using single particle luminescence," Journal of Physics, vol. 100, no. 5, Article ID 052038, 2008.

[65] T. Matsumoto, T. Ichimura, T. Yatsui, M. Kourogi, T. Saiki, and M. Ohtsu, "Fabrication of a near-field optical fiber probe with a nanometric metallized protrusion," Optical Review, vol. 5, no. 6, pp. 369-373, 1998.

[66] R. Eckert, J. M. Freyland, H. Gersen et al., "Near-field fluorescence imaging with $32 \mathrm{~nm}$ resolution based on microfabricated cantilevered probes," Applied Physics Letters, vol. 77, no. 23, pp. 3695-3697, 2000.

[67] T. Kalkbrenner, M. Ramstein, J. Mlynek, and V. Sandoghdar, "A single gold particle as a probe for apertureless scanning near-field optical microscopy," Journal of Microscopy, vol. 202, no. 1, pp. 72-76, 2001.

[68] H. G. Frey, S. Witt, K. Felderer, and R. Guckenberger, "Highresolution imaging of single fluorescent molecules with the optical near-field of a metal tip," Physical Review Letters, vol. 93, no. 20, Article ID 200801, 2004.

[69] J. N. Farahani, D. W. Pohl, H. J. Eisler, and B. Hecht, "Single quantum dot coupled to a scanning optical antenna: a tunable superemitter," Physical Review Letters, vol. 95, no. 1, Article ID 017402, pp. 1-4, 2005.

[70] T. H. Taminiau, R. J. Moerland, F. B. Segerink, L. Kuipers, and N. F. Van Hulst, " $\lambda / 4$ resonance of an optical monopole antenna probed by single molecule fluorescence," Nano Letters, vol. 7, no. 1, pp. 28-33, 2007.

[71] J. Dorfmüller, R. Vogelgesang, R. T. Weitz et al., "Fabry-Pérot resonances in one-dimensional plasmonic nanostructures," Nano Letters, vol. 9, no. 6, pp. 2372-2377, 2009.

[72] J. Dorfmüller, R. Vogelgesang, W. Khunsin, C. Rockstuhl, C. Etrich, and K. Kern, "Plasmonic nanowire antennas: experiment, simulation, and theory," Nano Letters, vol. 10, no. 9, pp. 3596-3603, 2010.

[73] M. Schnell, A. García-Etxarri, A. J. Huber, K. Crozier, J. Aizpurua, and R. Hillenbrand, "Controlling the near-field oscillations of loaded plasmonic nanoantennas," Nature Photonics, vol. 3, no. 5, pp. 287-291, 2009.

[74] P. Alonso-Gonzalez, M. Schnell, P. Sarriugarte et al., "Realspace mapping of Fano interference in plasmonic metamolecules," Nano Letters, vol. 11, no. 9, pp. 3922-3926, 2011.

[75] N. Félidj, G. Laurent, J. Grand et al., "Far-field raman imaging of short-wavelength particle plasmons on gold nanorods," Plasmonics, vol. 1, no. 1, pp. 35-39, 2006.

[76] G. Laurent, N. Félidj, J. Aubard et al., "Evidence of multipolar excitations in surface enhanced Raman scattering," Physical Review B, vol. 71, no. 4, pp. 1-6, 2005.

[77] C. Nobile, V. A. Fonoberov, S. Kudera et al., "Confined optical phonon modes in aligned nanorod arrays detected by resonant inelastic light scattering," Nano Letters, vol. 7, no. 2, pp. 476-479, 2007.

[78] M. Pelton, M. Liu, S. Park, N. F. Scherer, and P. Guyot-Sionnest, "Ultrafast resonant optical scattering from single gold nanorods: large nonlinearities and plasmon saturation," Physical Review B, vol. 73, no. 15, Article ID 155419, pp. 1-6, 2006.

[79] J. P. Hoogenboom, A. L. Lereu, and N. F. van Hulst, "Fabrication of molecular plasmonic devices with localized, subdiffraction-limit control over molecular positions," Patent. In press. 
[80] J. P. Hoogenboom, G. Sanchez-Mosteiro, G. C. Des Francs et al., "The single molecule probe: nanoscale vectorial mapping of photonic mode density in a metal nanocavity," Nano Letters, vol. 9, no. 3, pp. 1189-1195, 2009.

[81] A. Kinkhabwala, Z. Yu, S. Fan, Y. Avlasevich, K. Müllen, and W. E. Moerner, "Large single-molecule fluorescence enhancements produced by a bowtie nanoantenna," Nature Photonics, vol. 3, no. 11, pp. 654-657, 2009.

[82] R. P. Van Duyne, "Molecular plasmonics," Science, vol. 306, no. 5698, pp. 985-986, 2004.

[83] O. L. Muskens, V. Giannini, J. A. Sánchez-Gil, and J. Gómez Rivas, "Strong enhancement of the radiative decay rate of emitters by single plasmonic nanoantennas," Nano Letters, vol. 7, no. 9, pp. 2871-2875, 2007.

[84] G. P. Wiederrecht, J. E. Hall, and A. Bouhelier, "Control of molecular energy redistribution pathways via surface plasmon gating," Physical Review Letters, vol. 98, no. 8, Article ID 083001, 2007.

[85] O. G. Tovmachenko, C. Graf, D. J. Van Den Heuvel, A. Van Blaaderen, and H. C. Gerritsen, "Fluorescence enhancement by metal-core/silica-shell nanoparticles," Advanced Materials, vol. 18, no. 1, pp. 91-95, 2006.

[86] F. Tam, G. P. Goodrich, B. R. Johnson, and N. J. Halas, "Plasmonic enhancement of molecular fluorescence," Nano Letters, vol. 7, no. 2, pp. 496-501, 2007.

[87] Y. Fedutik, V. Temnov, U. Woggon, E. Ustinovich, and M. Artemyev, "Exciton-plasmon interaction in a composite metal-insulator-semiconductor nanowire system," Journal of the American Chemical Society, vol. 129, no. 48, pp. 1493914945, 2007.

[88] A. G. Curto, G. Volpe, T. H. Taminiau, M. P. Kreuzer, R. Quidant, and N. F. Van Hulst, "Unidirectional emission of a quantum dot coupled to a nanoantenna," Science, vol. 329, no. 5994, pp. 930-933, 2010.

[89] G. V. Hartland, M. Hu, O. Wilson, P. Mulvaney, and J. E. Sader, "Coherent excitation of vibrational modes in gold nanorods," Journal of Physical Chemistry B, vol. 106, no. 4, pp. 743-747, 2002.

[90] H. M. Chen, H. C. Peng, R. S. Liu et al., "Controlling the length and shape of gold nanorods," Journal of Physical Chemistry B, vol. 109, no. 42, pp. 19553-19555, 2005.

[91] P. K. Jain, S. Eustis, and M. A. El-Sayed, "Plasmon coupling in nanorod assemblies: optical absorption, discrete dipole approximation simulation, and exciton-coupling model," Journal of Physical Chemistry B, vol. 110, no. 37, pp. 1824318253, 2006.

[92] P. Mulvaney, J. Pérez-Juste, M. Giersig, L. M. Liz-Marzán, and C. Pecharromán, "Drastic surface plasmon mode shifts in gold nanorods due to electron charging," Plasmonics, vol. 1, no. 1, pp. 61-66, 2006.

[93] R. Zanella, A. Sandoval, P. Santiago, V. A. Basiuk, and J. M. Saniger, "New preparation method of gold nanoparticles on $\mathrm{SiO}_{2}$," Journal of Physical Chemistry B, vol. 110, no. 17, pp. 8559-8565, 2006.

[94] B. J. Wiley, Y. Chen, J. M. McLellan et al., "Synthesis and optical properties of silver nanobars and nanorice," Nano Letters, vol. 7, no. 4, pp. 1032-1036, 2007.

[95] T. Ito and S. Okazaki, "Pushing the limits of lithography," Nature, vol. 406, no. 6799, pp. 1027-1031, 2000.

[96] C. L. Haynes and R. P. Van Duyne, "Nanosphere lithography: a versatile nanofabrication tool for studies of size-dependent nanoparticle optics," Journal of Physical Chemistry B, vol. 105, no. 24, pp. 5599-5611, 2001.
[97] N. F. Van Hulst, J. A. Veerman, M. F. García-Parajó, and L. Kuipers, "Analysis of individual (macro)molecules and proteins using near-field optics," Journal of Chemical Physics, vol. 112, no. 18, pp. 7799-7810, 2000.

[98] J. Hernando, J. P. Hoogenboom, E. M. H. P. Van Dijk et al., "Single molecule photobleaching probes the exciton wave function in a multichromophoric system," Physical Review Letters, vol. 93, no. 23, Article ID 236404, 2004.

[99] J. P. Hoogenboom, E. M. H. P. Van Dijk, J. Hernando, N. F. Van Hulst, and M. F. García-Parajó, "Power-law-distributed dark states are the main pathway for photobleaching of single organic molecules," Physical Review Letters, vol. 95, no. 9, Article ID 097401, pp. 1-4, 2005.

[100] N. A. M. Verhaegh and A. Van Blaaderen, "Dispersions of rhodamine-labeled silica spheres: synthesis, characterization, and fluorescence confocal scanning laser microscopy," Langmuir, vol. 10, no. 5, pp. 1427-1438, 1994. 

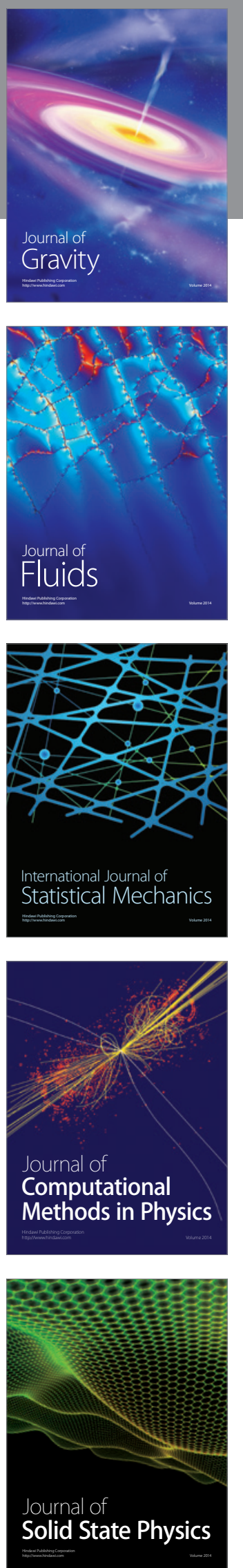

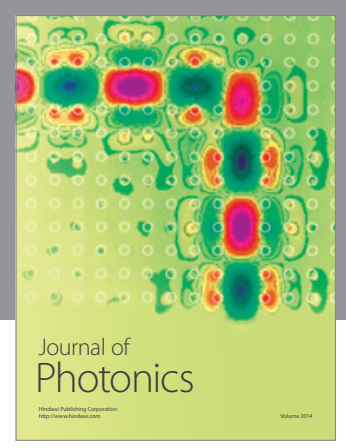

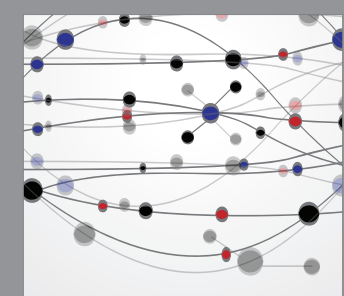

The Scientific World Journal
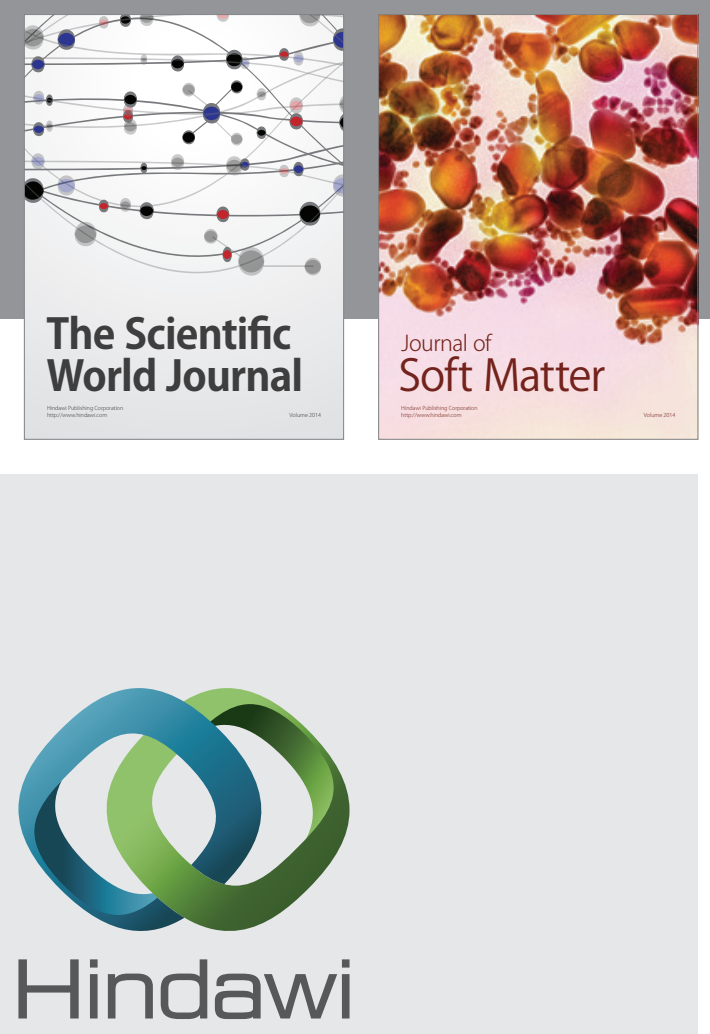

Submit your manuscripts at

http://www.hindawi.com
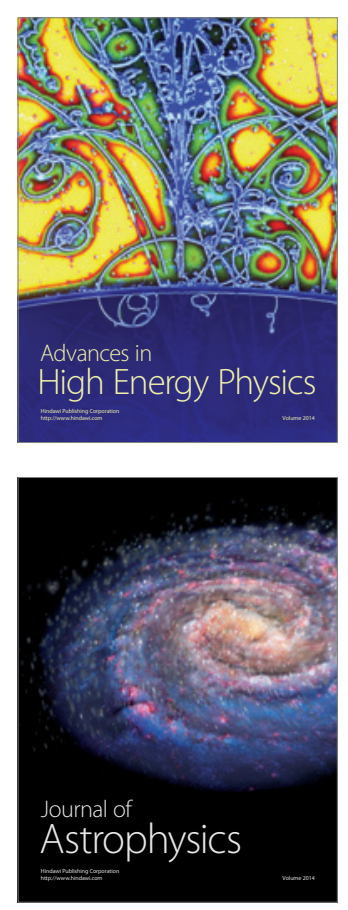
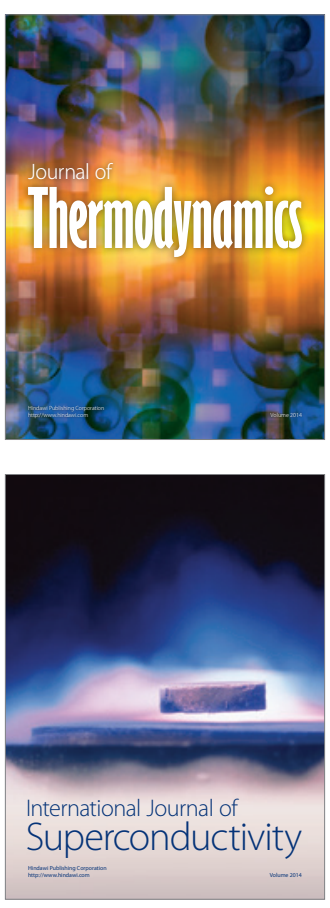
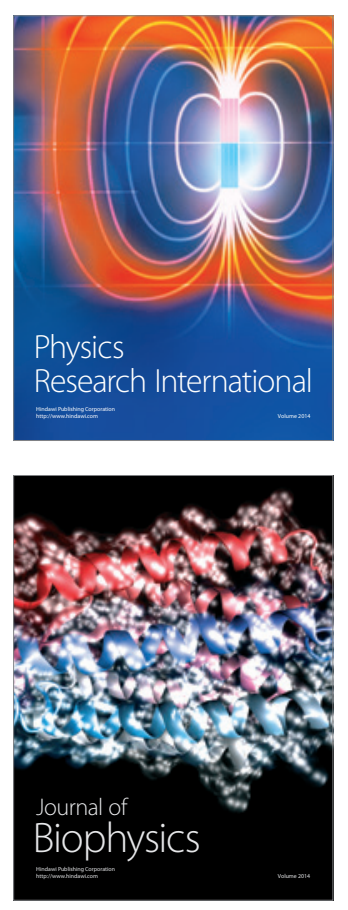
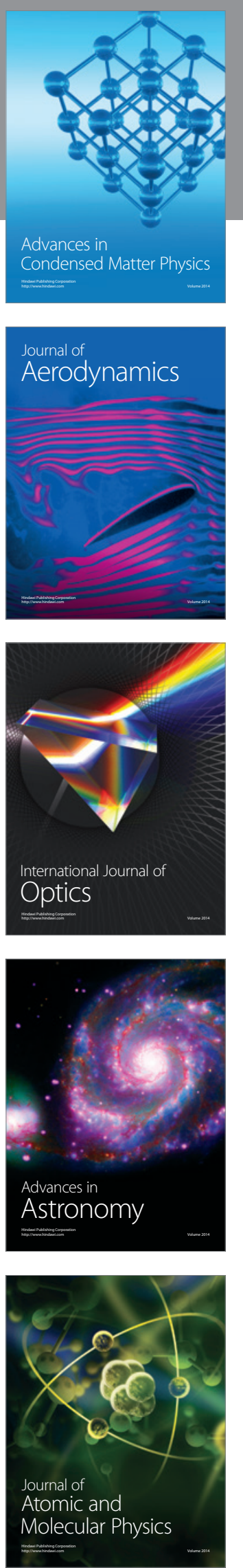